\title{
MINIMAL ROTATIONALLY INVARIANT BASES FOR HYPERELASTICITY*
}

\author{
GREGORY H. MILLER ${ }^{\dagger}$
}

\begin{abstract}
Rotationally invariant polynomial bases of the hyperelastic strain energy function are rederived using methods of group theory, invariant theory, and computational algebra. A set of minimal basis functions is given for each of the 11 Laue groups, with a complete set of rewriting syzygies. The ideal generated from this minimal basis agrees with the classic work of Smith and Rivlin [Trans. Amer. Math. Soc., 88 (1958), pp. 175-193]. However, the structure of the invariant algebra described here calls for fewer terms, beginning with the fourth degree in strain, for most groups.
\end{abstract}

Key words. elasticity, hyperelasticity, symmetry, integrity basis, Gröbner bases

AMS subject classifications. 74A20, 74B20, 13P10, 13A50, 14L24, 68W30

DOI. $10.1137 /$ S0036139903438776

1. Introduction. In 1958 Smith and Rivlin [20] derived a set of so-called integrity bases: a finite set of homogeneous polynomial functions of the strain, unique to each of 11 sets of symmetry groups (the Laue groups) which govern the symmetry of the strain energy function. These invariants were derived using theorems for the invariants of permutation groups (e.g., Weyl [25]). By "basis" it is meant that any arbitrary symmetry-invariant polynomial may be rewritten as a polynomial in these basis functions. Since the number of invariant homogeneous polynomials is unbounded, the discovery of a finite basis makes the problem of hyperelastic constitutive modeling tractable (and, indeed, far simpler than constructing a symmetry-invariant function as an expansion in symmetry-correct fourth- and higher-order elastic constant tensors). The integrity bases are particularly important for modeling time-dependent large deformation solid mechanics. Examples of computational methods requiring properly invariant hyperelastic descriptions include [15, 16, 14].

Since the important classification by Smith and Rivlin, a number of significant advances have been made in computational tools for algebra, particularly the theory of Gröbner bases, which has opened powerful new approaches to the study of group invariants (e.g., [24]). In this paper the elastic integrity bases are rederived using these new algorithmic approaches. The main point of this paper is to reexamine the invariant structure of hyperelastic materials using these modern methods. It will be shown that the integrity bases of Smith and Rivlin are correct in the sense that their integrity bases are finite bases which generate the correct invariant polynomial ideals. However, for most symmetry groups a number of syzygies exist which interrelate the invariant basis polynomials, and therefore their bases are not minimal (syzygies and minimality are described in this context in, e.g., [22]). It will be shown that for most groups, beginning at degree 4 in the Cauchy tensor (equivalently, the Lagrangian

* Received by the editors December 12, 2003; accepted for publication (in revised form) May 19, 2004; published electronically September 2, 2004. This work was sponsored by the U.S. Department of Energy (DOE) Mathematical, Information, and Computing Sciences Division contracts DE-AC0376 SF00098 and DE-FG02-03ER25579.

http://www.siam.org/journals/siap/64-6/43877.html

${ }^{\dagger}$ Department of Applied Science, University of California, 1 Shields Avenue, Davis, CA 95616 (grgmiller@ucdavis.edu) and Applied Numerical Algorithms Group, Lawrence Berkeley National Laboratory, 1 Cyclotron Road, Berkeley, CA 94720. 
strain tensor), the Smith and Rivlin integrity bases imply the existence of unnecessary polynomial terms.

In section 2 properties of the strain energy function are reviewed. This section sets the thermodynamic context for subsequent more mathematical sections and identifies the Cauchy tensor as the key fundamental variable controlling hyperelasticity. In section 3 the group theoretical properties of the 32 crystallographic point groups are described as they relate to the Cauchy tensor. Section 4 extends group theory to describe the algebraic structure of polynomial invariants of each group. Algebraic algorithms are described briefly in section 5 and used to construct a complete set of invariants. Simplifying relations among these invariants, syzygies are described in the appendix. A complete set of "rewriting syzygies" is presented, with which one could cast an arbitrary invariant polynomial into a minimal form. Select syzygies are also presented which demonstrate the algebraic dependence of "secondary invariants" upon the "primary invariants." Concluding remarks are made in section 6.

2. The strain energy function. The fundamental kinematic variable that governs hyperelasticity is the deformation tensor

$$
F_{\alpha \beta}=\frac{\partial x_{\alpha}}{\partial a_{\beta}},
$$

which describes the deformation of a spatial (Eulerian) frame $x$ with respect to a material (Lagrangian) frame $a$. The internal energy $\mathcal{E}$ is some function of the nine components of $F$, entropy $S$, and possibly other material constitutive parameters: $\mathcal{E}=\mathcal{E}(F, S)$.

Since the internal energy is a scalar function, its value must be independent of the reference frame of the observer. Thus, an observer utilizing a reference frame $\hat{x}$ will interpret the laboratory reference frame $x$ rotated through an arbitrary orthogonal rotation $Q\left(Q^{-1}=Q^{T}\right)$ and translated by an arbitrary vector $\hat{x}_{0}$ :

$$
\begin{aligned}
& \hat{x}=\hat{x}_{0}+Q x, \\
& \hat{F}=Q F .
\end{aligned}
$$

The possibly time-dependent translation $\hat{x}_{0}$ is independent of the material reference frame $\{a\}$ and therefore does not affect the observer's deformation tensor $\hat{F}$. In the observer's frame, the internal energy would be $\hat{\mathcal{E}}=\hat{\mathcal{E}}(Q F, S)$. And so, for the internal energy to be independent of the reference frame of the observer, the function must depend not on the components of $F$ individually but upon some combination of them that removes the dependence on $Q$.

One way of removing the $Q$-dependence is to factor $\hat{F}$ into a matrix of pure stretches and a matrix of rotations. The so-called right-polar decomposition of $F$ is

$$
F=R U,
$$

where $R$ is a rotation $\left(R^{-1}=R^{T}\right)$ and $U$ is symmetric. This decomposition is unique, with

$$
U^{T} U=F^{T} F=F^{T} Q^{T} Q F=\hat{F}^{T} \hat{F} .
$$

Instead of solving (2.5) for the six independent components of $U$, one might use directly the six independent components of $C$ - the Cauchy tensor (or "right CauchyGreen tensor")

$$
\begin{aligned}
C & =F^{T} F, \\
\mathcal{E} & =\mathcal{E}(C, S) .
\end{aligned}
$$


These manipulations determine the functional dependence of the internal energy in such a way as to make the result independent of the reference frame of an observer. The Cauchy tensor remains, however, dependent upon the orientation of the material with respect to the material reference frame $a$. For crystals with no rotational symmetry, this result is adequate, and one may without loss of generality construct hyperelastic equations of state (2.7) that are consistent with all symmetry constraints.

There are 230 space groups that classify the symmetry of single crystals. These are based upon 32 crystallographic point groups, which derive from consideration of rotations and reflections (reflections may also be referred to as improper $S_{1}$ rotations; collectively such operations will be called simply "rotations"), and become 230 upon consideration of translations consistent with the rotational symmetry. To discuss rotational invariance it is sufficient to consider the point groups. Of these 32 point groups, only two $\left(C_{1}\right.$ and $\left.C_{i}\right)$ are correctly modeled by $(2.7)$ without additional considerations of symmetry. The remaining 30 point groups classify materials which are symmetric with respect to certain discrete symmetry operations $\bar{\pi}$ on the atomic coordinates in the material reference frame:

$$
\begin{aligned}
\check{a} & =\bar{\pi}^{-1} a, \\
\check{F} & =F \bar{\pi}, \\
\check{C} & =\bar{\pi}^{T} C \bar{\pi} .
\end{aligned}
$$

For the internal energy to be invariant with respect to each of these discrete rotational mappings, one must have

$$
\mathcal{E}=\mathcal{E}(C, S)=\mathcal{E}\left(\bar{\pi}^{T} C \bar{\pi}, S\right) \quad \forall \bar{\pi} \in \bar{\Gamma}^{\bar{G}},
$$

where $\bar{\Gamma}^{\bar{G}}$ represents the set of rotation operations of the crystallographic point group $\bar{G}$ of the material (the symbols $\bar{\pi}, \bar{\Gamma}, \bar{G}$, etc. are used here to describe the group properties in the $\mathbb{R}^{3}$ coordinate space; the symbols $\pi, \Gamma, G$, etc. will denote the corresponding extension of these group properties to the $\mathbb{R}^{6}$ space of the unique Cauchy tensor elements).

3. Group theory. The crystallographic point groups may be described by a finite number of $3 \times 3$ matrices which rotate a vector, reflect it across a plane, or combinations thereof. The set $\bar{\Gamma}$ of these matrices $\bar{\pi}$ are a representation of a group algebra $\bar{G}$, which means (1) multiplication is associative, $\left(\bar{\pi}_{\alpha} \bar{\pi}_{\beta}\right) \bar{\pi}_{\gamma}=\bar{\pi}_{\alpha}\left(\bar{\pi}_{\beta} \bar{\pi}_{\gamma}\right)$ for each $\bar{\pi}_{\alpha}, \bar{\pi}_{\beta}, \bar{\pi}_{\gamma} \in \bar{\Gamma} ;(2)$ that for each $\bar{\pi}_{\alpha}, \bar{\pi}_{\beta} \in \bar{\Gamma}$, the product $\bar{\pi}_{\alpha} \bar{\pi}_{\beta}$ is also contained in $\bar{\Gamma}$; (3) there exists an identity $\bar{E} \in \bar{\Gamma}$ such that $\bar{E} \bar{\pi}_{\alpha}=\bar{\pi}_{\alpha} \bar{E}=\bar{\pi}_{\alpha}$ for each $\bar{\pi}_{\alpha} \in \bar{\Gamma}$; and (4) for each $\bar{\pi}_{\alpha} \in \bar{\Gamma}$, there exists an inverse $\bar{\pi}_{\alpha}^{-1}$ such that $\bar{\pi}_{\alpha} \bar{\pi}_{\alpha}^{-1}=\bar{E}$.

One may use the property (2.10) to construct a set of $6 \times 6$ matrix operators $\pi_{1}$

$$
\pi_{1}=\left(\begin{array}{cccccc}
\bar{\pi}_{11}^{2} & \bar{\pi}_{21}^{2} & \bar{\pi}_{31}^{2} & 2 \bar{\pi}_{31} \bar{\pi}_{21} & 2 \bar{\pi}_{11} \bar{\pi}_{31} & 2 \bar{\pi}_{11} \bar{\pi}_{21} \\
\bar{\pi}_{12}^{2} & \bar{\pi}_{22}^{2} & \bar{\pi}_{32}^{2} & 2 \bar{\pi}_{22} \bar{\pi}_{32} & 2 \bar{\pi}_{32} \bar{\pi}_{12} & 2 \bar{\pi}_{22} \bar{\pi}_{12} \\
\bar{\pi}_{13}^{2} & \bar{\pi}_{23}^{2} & \bar{\pi}_{33}^{2} & 2 \bar{\pi}_{33} \bar{\pi}_{23} & 2 \bar{\pi}_{33} \bar{\pi}_{13} & 2 \bar{\pi}_{23} \bar{\pi}_{13} \\
\bar{\pi}_{12} \bar{\pi}_{13} & \bar{\pi}_{22} \bar{\pi}_{23} & \bar{\pi}_{32} \bar{\pi}_{33} & \bar{\pi}_{22} \bar{\pi}_{33}+\bar{\pi}_{32} \bar{\pi}_{23} & \bar{\pi}_{12} \bar{\pi}_{33}+\bar{\pi}_{32} \bar{\pi}_{13} & \bar{\pi}_{12} \bar{\pi}_{23}+\bar{\pi}_{22} \bar{\pi}_{13} \\
\bar{\pi}_{11} \bar{\pi}_{13} & \bar{\pi}_{21} \bar{\pi}_{23} & \bar{\pi}_{31} \bar{\pi}_{33} & \bar{\pi}_{21} \bar{\pi}_{33}+\bar{\pi}_{31} \bar{\pi}_{23} & \bar{\pi}_{11} \bar{\pi}_{33}+\bar{\pi}_{31} \bar{\pi}_{13} & \bar{\pi}_{11} \bar{\pi}_{23}+\bar{\pi}_{21} \bar{\pi}_{13} \\
\bar{\pi}_{11} \bar{\pi}_{12} & \bar{\pi}_{21} \bar{\pi}_{22} & \bar{\pi}_{31} \bar{\pi}_{32} & \bar{\pi}_{21} \bar{\pi}_{32}+\bar{\pi}_{31} \bar{\pi}_{22} & \bar{\pi}_{11} \bar{\pi}_{32}+\bar{\pi}_{31} \bar{\pi}_{12} & \bar{\pi}_{11} \bar{\pi}_{22}+\bar{\pi}_{21} \bar{\pi}_{12}
\end{array}\right)
$$


that transform the six-dimensional vector $\eta=\left(C_{11}, C_{22}, C_{33}, C_{23}\left(=C_{32}\right), C_{13}\left(=C_{31}\right)\right.$, $\left.C_{12}\left(=C_{21}\right)\right)^{T}$, or in Voigt notation $\left(C_{1}, C_{2}, C_{3}, C_{4}, C_{5}, C_{6}\right)^{T}$, according to

$$
\check{\eta}=\pi_{1} \eta \text {. }
$$

In the language of Murnaghan [18, Ch. 3], the matrices $\pi_{1}$ are symmetrized Kronecker products of the transformation matrices $\bar{\pi}^{T}$. The $d$-form matrices $\pi_{d}$ introduced below are symmetrized Kronecker $d$-powers of the transformations $\pi_{1}$.

Note that the set $\Gamma$ of matrices $\pi_{1}$, formed from the elements $\bar{\pi} \in \bar{\Gamma}$ of group $\bar{G}$, define a group algebra $G$ that may be different from $\bar{G}$ (e.g., [8]). In particular, the transformation matrices (3.1) effectively introduce inversion symmetry where none may have existed in the original group. Thus, as with Laue diffraction, the 32 crystallographic point groups reduce immediately to the 11 Laue groups.

A linear combination $\kappa$ of elements of the Cauchy tensor is invariant to all symmetry operations if for each $\pi_{1} \in \Gamma$ one has $\kappa=\pi_{1} \kappa$; thus $\kappa$ must be an eigenvector of each matrix $\pi_{1}$ with eigenvalue 1 . Or

$$
\kappa=\mathcal{P}_{R} \kappa,
$$

with

$$
\mathcal{P}_{R}=\frac{1}{|\Gamma|} \sum_{\pi_{1} \in \Gamma} \pi_{1}
$$

and $|\Gamma|$ the cardinality of the group. $\mathcal{P}_{R}$ is the Reynolds operator, a special case of the more general symmetry projection operator which projects a vector onto an irreducible representation of the group (e.g., $[6$, Ch. 6]). The Reynolds operator projects a vector onto the unique totally symmetric representation. $\mathcal{P}_{R}$ is a projection, $\mathcal{P}_{R}{ }^{2}=\mathcal{P}_{R}$, by virtue of the property of groups that $\pi_{1 \alpha} \Gamma=\Gamma$ for all $\pi_{1 \alpha} \in \Gamma$. Consequently, the eigenvalues of $\mathcal{P}_{R}$ are all either 0 or 1 . And, therefore, the number $N_{1}$ of linearly independent degree-1 invariant vectors is given by the number of unity eigenvalues of $\mathcal{P}_{R}$, which is equal to the trace of $\mathcal{P}_{R}$ :

$$
N_{1}=\operatorname{trace}\left(\mathcal{P}_{R}\right)=\frac{1}{|\Gamma|} \sum_{\pi_{1} \in \Gamma} \operatorname{trace}\left(\pi_{1}\right) .
$$

To evaluate this equation for any group, one tabulates the symmetry operations by type (Table 3.1 displays the operations of each group and their assumed orientation with respect to the assumed orthogonal Lagrangian coordinate system $a$ ). The numbers of symmetry operations $\pi_{1}$, by type and group $\Gamma$, are given in Table 3.2. The traces may be calculated from the eigenvalues listed in Table 3.3.

Invariants of higher degree lie in the $\left(\begin{array}{c}6+d-1 \\ d\end{array}\right)$-dimensional space formed by the unique combinations of degree- $d$ monomials (e.g., a basis for degree- 2 monomials is given by the 21 homogeneous terms $C_{i} C_{j \geq i}$ in a process analogous to that described by (3.1)). From the matrices $\pi_{1}$, so-called $d$-form matrices $\pi_{d}$ may be constructed easily to represent the action of the symmetry operations on the degree- $d$ terms. The number $N_{d}$ of linearly independent degree- $d$ symmetry-invariant terms are constructed as in the degree- 1 case with

$$
\mathcal{P}_{R, d}=\frac{1}{|\Gamma|} \sum_{\pi_{d} \in \Gamma} \pi_{d}
$$


TABLE 3.1

Settings for those crystallographic point groups with planes and axes (Wulff stereographic projections). Bold lines are mirror planes. Open and closed circles are general positions, above and below plane $z=0$, respectively. Closed symbols with $n$-fold symmetry are rotation axes, and mixed open-closed symbols with n-fold symmetry are improper rotations.
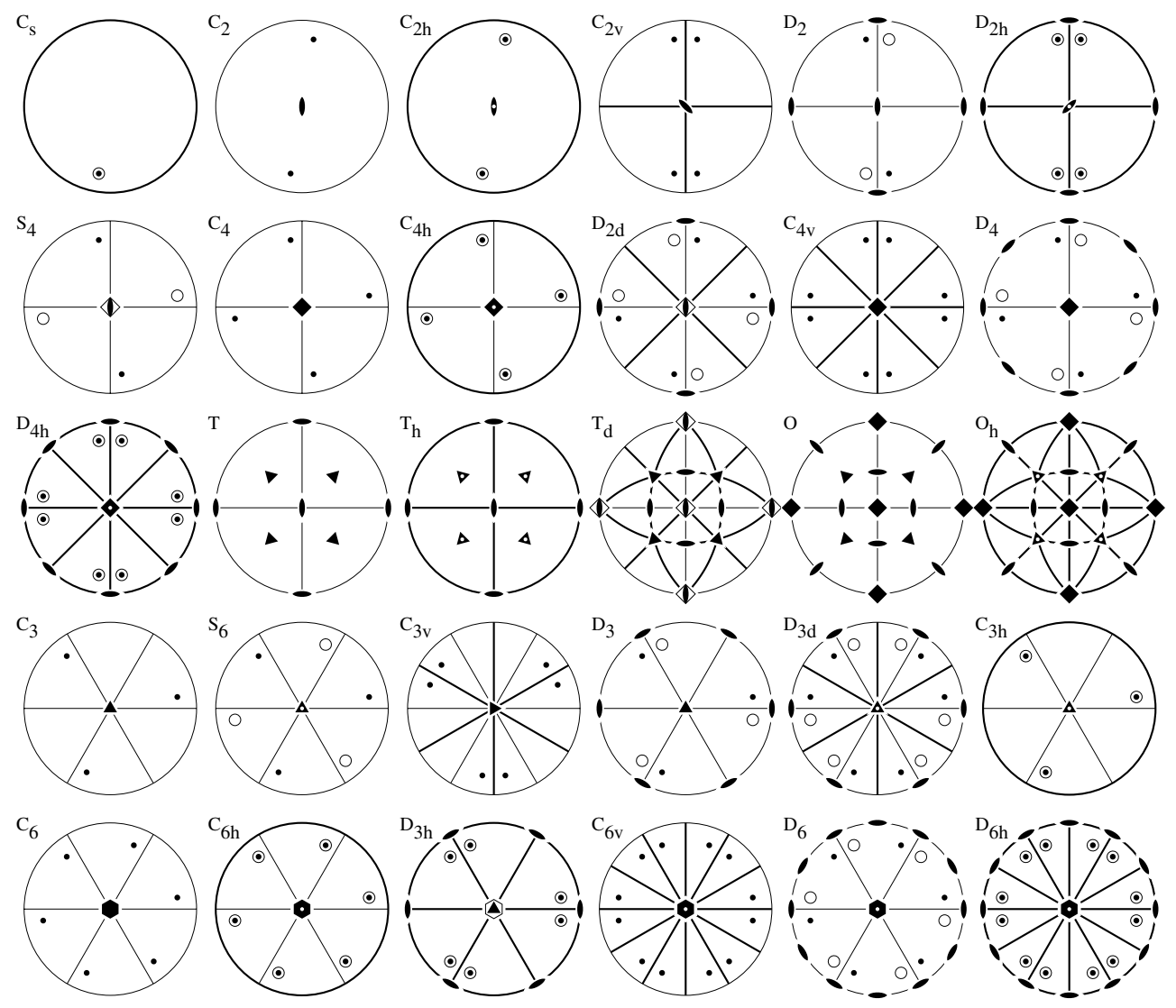

TABLE 3.2

Number of distinct occurrences of operations $\pi$ in the crystallographic point groups, in the $\mathbb{R}^{6}$ space of the Cauchy tensor. The symbols used here are Schoenflies notation: $E$ is the identity, I is inversion on all three orthogonal axes, $C_{n}$ is an $n$-fold rotation, $S_{n}$ is an improper $n$-fold rotation, and $\sigma=S_{1}$ is a mirror reflection.

\begin{tabular}{lllllll} 
Group $\Gamma$ & $|\Gamma|$ & $E, I$ & $C_{2}, \sigma$ & $C_{3}, S_{6}$ & $C_{4}, S_{4}$ & $C_{6}, S_{3}$ \\
\hline$C_{1}, C_{i}$ & 1 & 1 & & & & \\
$C_{s}, C_{2}, C_{2 h}$ & 2 & 1 & 1 & & & \\
$C_{2 v}, D_{2}, D_{2 h}$ & 4 & 1 & 3 & & & \\
$S_{4}, C_{4}, C_{4 h}$ & 4 & 1 & 1 & & 2 & \\
$D_{2 d}, C_{4 v}, D_{4}, D_{4 h}$ & 8 & 1 & 5 & & 2 & \\
$T, T_{h}$ & 12 & 1 & 3 & 8 & & \\
$T_{d}, O, O_{h}$ & 24 & 1 & 9 & 8 & 6 & \\
$C_{3}, S_{6}$ & 3 & 1 & & 2 & & \\
$C_{3 v}, D_{3}, D_{3 d}$ & 6 & 1 & 3 & 2 & & 2 \\
$C_{3 h}, C_{6}, C_{6 h}$ & 6 & 1 & 1 & 2 & & 2 \\
$D_{3 h}, C_{6 v}, D_{6}, D_{6 h}$ & 12 & 1 & 7 & 2 & & 2 \\
\hline
\end{tabular}


TABLE 3.3

Eigenvalues of the point group operators $\pi$ in the space $\mathbb{R}^{6}$ of the Cauchy tensor.

\begin{tabular}{lllllllc}
\multicolumn{7}{l}{ Operator $\pi_{1}$} & \multicolumn{7}{l}{ Eigenvalues } \\
\hline$E$ & $I$ & 1 & 1 & 1 & 1 & 1 & 1 \\
$C_{2}$ & $\sigma$ & 1 & 1 & 1 & 1 & -1 & -1 \\
$C_{3}$ & $S_{6}$ & 1 & 1 & $e^{\frac{2 \pi i}{3}}$ & $e^{\frac{2 \pi i}{3}}$ & $e^{-\frac{2 \pi i}{3}}$ & $e^{-\frac{2 \pi i}{3}}$ \\
$C_{4}$ & $S_{4}$ & 1 & 1 & -1 & -1 & $e^{\frac{\pi i}{2}}$ & $e^{-\frac{\pi i}{2}}$ \\
$C_{6}$ & $S_{3}$ & 1 & 1 & $e^{\frac{\pi i}{3}}$ & $e^{-\frac{\pi i}{3}}$ & $e^{\frac{2 \pi i}{3}}$ & $e^{-\frac{2 \pi i}{3}}$ \\
\hline
\end{tabular}

$$
N_{d}=\operatorname{trace}\left(\mathcal{P}_{R, d}\right)=\frac{1}{|\Gamma|} \sum_{\pi_{d} \in \Gamma} \operatorname{trace}\left(\pi_{d}\right)
$$

In practice it is not necessary to actually create the matrices $\pi_{d}$. The eigenvalues of the $d$-form matrices $\pi_{d}$ are $\lambda_{1}^{d_{1}} \cdots \lambda_{6}^{d_{6}}$, with $\lambda_{i}$ representing the $i$ th eigenvalue of $\pi_{1}$, and with the exponents $d_{i}$ subject to $d_{1}+\cdots+d_{6}=d$. Therefore,

$$
\operatorname{trace}\left(\pi_{d}\right)=\sum_{d_{1}+\cdots+d_{6}=d} \lambda_{1}^{d_{1}} \cdots \lambda_{6}^{d_{6}} \text {. }
$$

For completeness, one has also the scalar degree-0 term: the number "1." This polynomial invariant of degree 0 is generated by $\pi_{0}=1$, whence $N_{0}=1$.

Via the projections $\mathcal{P}_{R, d}$ group theory provides a method for the construction of all linearly independent degree- $d$ symmetry-invariant homogeneous polynomials. The number of such polynomials is unbounded, however, since for any degree $d$ the number of terms is at least as large as $\left(\begin{array}{c}N_{1}+d-1 \\ d\end{array}\right)$ - the number of distinct degree- $d$ polynomials formed from by multiplying together different combinations of degree- 1 polynomials.

A finite polynomial basis, a set of invariant polynomials from which all others may be constructed, exists. The number of terms in this basis and some properties of it are provided by theorems of invariant theory described below. The construction of the actual invariant polynomial bases is accomplished with the Gröbner basis methods of computational algebra, described in the subsequent section.

4. Invariant theory. The Hilbert series $\Phi(z)$ (also known as Poincaré's series) is a polynomial in the dummy variable $z$ where the coefficient of $z^{n}$ is the number of polynomial invariants of degree $n$. Spencer's generating functions [21], used in the study of invariants in continuum mechanics, are particular instances of the Hilbert series constructed by different means than those employed here. Following Sturmfels [24, Theorem 2.2.1] after [17],

$$
\begin{aligned}
\Phi(z) & =\sum_{d=0}^{\infty} N_{d} z^{d}=\frac{1}{|\Gamma|} \sum_{\pi_{1} \in \Gamma} \sum_{d=0}^{\infty} \sum_{d_{1}+\cdots+d_{6}=d} \lambda_{1}^{d_{1}} \cdots \lambda_{6}^{d_{6}} z^{d}, \\
& =\frac{1}{|\Gamma|} \sum_{\pi_{1} \in \Gamma} \sum_{d_{1}, \ldots, d_{6}=0}^{\infty} \lambda_{1}^{d_{1}} \cdots \lambda_{6}^{d_{6}} z^{d_{1}+\cdots+d_{6}} \\
& =\frac{1}{|\Gamma|} \sum_{\pi_{1} \in \Gamma} \prod_{n=1}^{6}\left(1+\left(\lambda_{n} z\right)+\left(\lambda_{n} z\right)^{2}+\cdots\right) \\
& =\frac{1}{|\Gamma|} \sum_{\pi_{1} \in \Gamma} \prod_{n=1}^{6} \frac{1}{\left(1-\lambda_{n} z\right)}=\frac{1}{|\Gamma|} \sum_{\pi_{1} \in \Gamma} \frac{1}{\operatorname{det}\left(I-\pi_{1} z\right)},
\end{aligned}
$$

where $\lambda_{\alpha}=\lambda_{\alpha}\left(\pi_{1}\right)$, and where one assumes that $|z|<1$. 
TABLE 4.1

Contributions of point group operators to the Hilbert series.

\begin{tabular}{lc} 
Operation $\pi_{1}$ & Hilbert series contribution \\
\hline$E, I$ & $\frac{1}{(1-z)^{6}}$ \\
$C_{2}, \sigma$ & $\frac{1}{(1-z)^{4}(1+z)^{2}}=\frac{1}{(1-z)^{2}\left(1-z^{2}\right)^{2}}$ \\
$C_{3}, S_{6}$ & $\frac{1}{(1-z)^{2}\left(1+z+z^{2}\right)^{2}}=\frac{1}{\left(1-z^{3}\right)^{2}}$ \\
$C_{4}, S_{4}$ & $\frac{1}{\left(1-z^{2}\right)^{2}\left(1+z^{2}\right)}=\frac{1}{\left(1-z^{2}\right)\left(1-z^{4}\right)}$ \\
$C_{6}, S_{3}$ & $\frac{1}{(1-z)^{2}\left(1-z+z^{2}\right)\left(1+z+z^{2}\right)}=\frac{(1+z)}{(1-z)\left(1-z^{6}\right)}$ \\
\hline
\end{tabular}

By means of this result, it is apparent that the Hilbert series for a given crystallographic point group may be algebraically constructed by summing factors $1 / \operatorname{det}(I-$ $\left.\pi_{1} z\right)$ corresponding to the individual operators $\pi_{1}$ that occur in the point group. These factors are summarized in Table 4.1.

One may then construct the Hilbert series according to the formula

$$
\begin{aligned}
\Phi_{\Gamma}(z)=\frac{1}{|\Gamma|}[ & \frac{N(E, I)}{(1-z)^{6}}+\frac{N\left(C_{2}, \sigma\right)}{(1-z)^{2}\left(1-z^{2}\right)^{2}}+\frac{N\left(C_{3}, S_{6}\right)}{\left(1-z^{3}\right)^{2}} \\
& \left.+\frac{N\left(C_{4}, S_{4}\right)}{\left(1-z^{2}\right)\left(1-z^{4}\right)}+\frac{N\left(C_{6}, S_{3}\right)(1+z)}{(1-z)\left(1-z^{6}\right)}\right]
\end{aligned}
$$

where $N(\pi, \ldots)$ is the number of occurrences of symmetry operators $\pi, \ldots \in \Gamma$, tabulated for the crystallographic point groups in Table 3.2.

Finite groups have the Cohen-Macaulay property of commutative algebra (e.g., [23]), which has significance for this project in that it implies that certain important properties of the invariant group algebra may be deduced by appropriate factorizations of the Hilbert series, called "Molien functions" or "Hironaka decompositions":

$$
\Phi(z)=\frac{\sum_{i=0}^{t-1} z^{e_{i}}}{\prod_{j=1}^{n}\left(1-z^{d_{j}}\right)} .
$$

The interpretation of these factorizations is that there exist $n$ primary invariants $\theta$ which comprise a "homogeneous system of parameters" (HSOP), with degrees $d_{i}=$ $\operatorname{deg}\left(\theta_{i}\right)$, and where $n$ is the number of variables (six for the Cauchy tensor elements). These are represented in the denominator of the Molien function. Since a factor $1 /$ $\left(1-z^{d}\right)$ contributes (multiplicatively) $1+z^{d}+z^{2 d}+z^{3 d}+\cdots$ to the Hilbert series, these factors are unrestricted in a polynomial representation. The numerator represents the $t$ secondary invariants $\phi$, with degrees $e_{j}=\operatorname{deg}\left(\phi_{j}\right)$ and cardinality

$$
t=\frac{1}{|\Gamma|} \prod_{i=1}^{n} d_{i},
$$

including the degree- 0 term " 1 ." Since factors $z^{e}$ in the numerator contribute only $z^{e}$ (multiplicatively) to the Hilbert series, the implication is that these factors occur at most once in a polynomial representation. 
TABLE 4.2

Molien factorizations of the Hilbert series of crystallographic point groups in the $\mathbb{R}^{6}$ space of the Cauchy tensor.

\begin{tabular}{|c|c|}
\hline Groups $\Gamma$ & Molien function \\
\hline $\begin{array}{l}\text { triclinic } \\
C_{1}, C_{i}\end{array}$ & $\frac{1}{(1-z)^{6}}$ \\
\hline $\begin{array}{l}\text { monoclinic } \\
C_{s}, C_{2}, C_{2 h}\end{array}$ & $\frac{1+z^{2}}{(1-z)^{4}\left(1-z^{2}\right)^{2}}$ \\
\hline $\begin{array}{l}\text { orthorhombic } \\
C_{2 v}, D_{2}, D_{2 h}\end{array}$ & $\frac{1+z^{3}}{(1-z)^{3}\left(1-z^{2}\right)^{3}}$ \\
\hline $\begin{array}{l}\text { tetragonal } \\
S_{4}, C_{4}, C_{4 h}\end{array}$ & $\frac{1+z^{2}+4 z^{3}+z^{4}+z^{6}}{(1-z)^{2}\left(1-z^{2}\right)^{3}\left(1-z^{4}\right)}$ \\
\hline $\begin{array}{l}\text { tetragonal } \\
D_{2 d}, C_{4 v}, D_{4}, D_{4 h}\end{array}$ & $\frac{1+2 z^{3}+z^{6}}{(1-z)^{2}\left(1-z^{2}\right)^{3}\left(1-z^{4}\right)}$ \\
\hline $\begin{array}{l}\text { cubic } \\
T, T_{h}\end{array}$ & $\frac{1+3 z^{3}+2 z^{4}+2 z^{5}+3 z^{6}+z^{9}}{(1-z)\left(1-z^{2}\right)^{2}\left(1-z^{3}\right)^{2}\left(1-z^{4}\right)}$ \\
\hline $\begin{array}{l}\text { cubic } \\
T_{d}, O, O_{h}\end{array}$ & $\frac{1+z^{3}+z^{4}+z^{5}+z^{6}+z^{9}}{(1-z)\left(1-z^{2}\right)^{2}\left(1-z^{3}\right)^{2}\left(1-z^{4}\right)}$ \\
\hline $\begin{array}{l}\text { trigonal } \\
C_{3}, S_{6}\end{array}$ & $\frac{1+2 z^{2}+6 z^{3}+2 z^{4}+z^{6}}{(1-z)^{2}\left(1-z^{2}\right)^{2}\left(1-z^{3}\right)^{2}}$ \\
\hline $\begin{array}{l}\text { trigonal } \\
C_{3 v}, D_{3}, D_{3 d}\end{array}$ & $\frac{1+z^{2}+2 z^{3}+z^{4}+z^{6}}{(1-z)^{2}\left(1-z^{2}\right)^{2}\left(1-z^{3}\right)^{2}}$ \\
\hline $\begin{array}{l}\text { hexagonal } \\
C_{3 h}, C_{6}, C_{6 h}\end{array}$ & $\frac{1+3 z^{3}+2 z^{4}+2 z^{5}+3 z^{6}+z^{9}}{(1-z)^{2}\left(1-z^{2}\right)^{2}\left(1-z^{3}\right)\left(1-z^{6}\right)}$ \\
\hline $\begin{array}{l}\text { hexagonal } \\
D_{3 h}, C_{6 v}, D_{6}, D_{6 h}\end{array}$ & $\frac{1+z^{3}+z^{4}+z^{5}+z^{6}+z^{9}}{(1-z)^{2}\left(1-z^{2}\right)^{2}\left(1-z^{3}\right)\left(1-z^{6}\right)}$ \\
\hline
\end{tabular}

A consequence of the Molien function is that all symmetry-invariant polynomial functions $P$ of the Cauchy tensor may be expressed in the form

$$
P(\{\theta\},\{\phi\})=\sum_{\alpha=0}^{t-1} \phi_{\alpha} P_{\alpha}(\{\theta\})
$$

where $P_{\alpha}(\{\theta\})$ is an arbitrary polynomial in the primary invariants, and where each secondary invariant $\phi_{\alpha}$ occurs at most once. For all groups henceforth let $\phi_{0}=1$, and consider only nontrivial secondary invariants.

Molien factorizations of the crystallographic point groups, constructed using (4.2) for the invariants of the Cauchy tensor terms, are given in Table 4.2. These functions are fully reduced in that there is no common algebraic factor to both numerator and denominator, and in this sense the implied size of the invariant set is minimal. These factorizations are not unique. For example, the factorization displayed for group $C_{2 v}$ implies primary invariants of degree $1,1,1,2,2,2$ and one nontrivial secondary invariant of degree 3 . However, multiplication of numerator and denominator by $(1+z)$ gives the function 


$$
\frac{1+z+z^{2}+z^{4}}{(1-z)^{2}\left(1-z^{2}\right)^{4}},
$$

implying primary invariants of degree $1,1,2,2,2,2$ and three nontrivial secondary invariants of degree $1,2,4$. If the form given in Table 4.2 exists, then the alternative form (4.6) is not minimal. The question of existence must be settled by constructing the algebraically independent basis functions of the group and partitioning them into primary and secondary invariants (e.g., [19, p. 101]). It will be shown that HSOPs of the degrees indicated by the reduced functions in Table 4.2 exist.

Since secondary $\phi_{\alpha}$ is an invariant, so is $\phi_{\alpha}^{2}$. However, $\phi_{\alpha}^{2}$ is not represented in the Hilbert series. Thus, the invariant $\phi_{\alpha}^{2}$ must be expressible by some polynomial of the form (4.5). This implies the existence of syzygies - polynomial equalities that relate the secondary and primary invariants. A set of syzygies may be found to serve as "rewriting rules" for systematic conversion of a general polynomial $P(\{\theta\},\{\phi\})$ into the minimal form given by the right-hand side of (4.5).

An HSOP is a minimal set of algebraically independent polynomials, with cardinality 6 , equal to the number of independent variables in $C$. There cannot be more algebraically independent homogeneous polynomials, and so any additional polynomial (in particular, the secondary invariants) must possess an algebraic dependence upon the primary invariants. The algebraic relations expressing a particular secondary invariant in terms of the primary ones are also expressible as syzygies.

The six primary invariants of each crystal point group are therefore algebraic (vs. polynomial) invariants; all polynomial invariants are expressible as algebraic functions of the primary invariants. This settles a conundrum regarding the number of degrees of freedom. The elastic Cauchy tensor has six degrees of freedom, and there are six algebraic invariants. The additional apparent degrees of freedom represented by the number of secondary invariants (aside from the trivial one, 1, of degree zero) are a consequence of assuming a polynomial form for the invariant energy function.

5. Computational algebra: Gröbner bases. In $\mathbb{R}^{3}$, the space of material coordinates $a$, and in $\mathbb{R}^{6}$, the space of Cauchy tensor components, some matrix operations of the groups (e.g., corresponding to rotations through $2 \pi / 3$ ) contain factors of $\sqrt{3} / 2$. However, polynomials generated through the Reynolds operator contain only integer coefficients, so it is sufficient to study the properties of $\mathbb{Q}[C]$, the ring over rational numbers $\mathbb{Q}$ of polynomials in the Cauchy tensor elements $C$. Let $F=\left\{f_{i} \mid f_{i} \in \mathbb{Q}[C], f_{i}=\mathcal{P}_{R} f_{i}\right\}$ be some set of invariant polynomials. The ideal generated by $F, I(F)$ is the set of all polynomials $p_{1} f_{1}+p_{2} f_{2}+\cdots, p_{i} \in \mathbb{Q}[C]$, that are dependent on elements of $F$; i.e., $g \in I(F)$ and $h \in \mathbb{Q}[C]$ implies that $g h \in I(F)$, and $g, f \in I(F)$ implies that $g+f \in I(F)$. The objective is to construct the smallest basis $F$ consisting of homogeneous invariant polynomials, with degrees consistent with the Molien function of the group, such that $I(F)$ is equal to the complete invariant ideal $I\left(\mathbb{Q}[C]^{G}\right)$ (see, e.g., $[23,4]$ and $[3]$ for algebra concepts, and the latter also for Gröbner bases).

Algorithms designed to address this problem require the capability of deciding whether some polynomial $f$ is in the ideal $I(F)$. The solution is to construct a special basis $\mathcal{G B}(F)$, the Gröbner basis, with $I(\mathcal{G B}(F))=I(F)$. That is, $\mathcal{G B}$ is an alternative basis that generates the same ideal as $F$. The key property of a Gröbner basis is that for any $f \in I(F), f \rightarrow_{\mathcal{G B}} 0: f$ is reducible to zero by successive steps of a Euclidean reduction algorithm. The reduction property is linked to a notion of term order; a unique reduced Gröbner is specified by the basis $F$, and a specification of the term order $\succ$. 
Group theory shows how, using the Reynolds operator, invariant polynomials may be generated, and from the Molien function one has an idea of what the degrees of primary and secondary invariants may be. Given a set of six homogeneous polynomial functions with degrees that are compatible with their being primary invariants, the first task is to show whether or not they are an HSOP. An algorithm for this task is given by Sturmfels [24, algorithm 2.5.3]. First, one uses the Reynolds operator ${ }^{1}$ to generate a set of homogeneous polynomial invariants $\theta^{\prime}(C)$ of the Cauchy tensor elements. Next, construct a polynomial basis set $F=\left\{\theta_{1}^{\prime}-y_{1}, \ldots, \theta_{6}^{\prime}-y_{6}\right\}$ in the variables $C$ and new slack variables $y$, with lexicographic order $C_{1}>\cdots>C_{6}>$ $y_{1}>\cdots>y_{6}$. This is an "elimination order" that systematically eliminates terms in $C$ from the head of each polynomial in the basis during construction of the Gröbner basis (the head term is the greatest with respect to the specified order-lexicographic in this case). Generate the Gröbner basis $\mathcal{G B}(F)$. Let $\mathcal{G} \mathcal{B}^{\prime}=\mathcal{G B}(F) \cap \mathbb{Q}[y]$ be the set of polynomials found in $\mathcal{G B}(F)$ containing only variables $y$. If $\mathcal{G B}^{\prime}=\emptyset$, then $\left\{\theta^{\prime}\right\}$ are algebraically independent; they may be chosen to comprise the HSOP of the group. If $\mathcal{G} \mathcal{B}^{\prime} \neq \emptyset$, then the functions contained in $\mathcal{G B}^{\prime}$ represent polynomial equations $P\left(y_{1}, y_{2}, \ldots, y_{6}\right)=0$ which represent syzygies among the variables $y$ hence amongst the functions $\theta^{\prime}$. This property will be exploited to determine syzygies.

The second task is the determination of secondary invariant polynomials [24, algorithm 2.5.14]. Begin with $F=\{\theta\}$, the set of primary invariants, and let $\phi=\emptyset$ be the set of discovered secondary invariants. Compute $\mathcal{G B}=\mathcal{G B}(F)$ with respect to any valid term order. For each degree indicated in the numerator of the Molien function, use the Reynolds operator to construct a set of linearly independent homogeneous invariants. Those candidate polynomials $\phi^{\prime}$ that reduce to zero with $\mathcal{G B}$ have a polynomial dependence on $\{\theta\}$ and are not valid secondary invariants. Those $\phi^{\prime}$ that do not reduce to zero are secondary invariants; $\phi:=\phi \cup\left\{\phi^{\prime}\right\}$.

To deduce rewriting syzygies, i.e., syzygies of the form $\phi_{i} \phi_{j}=p_{0}+\sum_{k} p_{k} \phi_{k}$, $p_{i} \in \mathbb{Q}[\theta]$, another algorithm based on Gröbner bases has been proposed [24, algorithm 2.5.6]. One computes the Gröbner basis of $F=\left\{\theta_{1}-y_{1}, \ldots, \theta_{6}-y_{6}, \phi_{1}-z_{1} \ldots\right\}$ in the variables $C$ and slack variables $y$ and $z$. Sturmfels recommends the variable order $C_{1}>\cdots>C_{6}>y_{1}>\cdots>y_{6}>z_{1}>\cdots$ and suggests the following term order $\succ$. Term $C^{\alpha} y^{\beta} z^{\gamma} \succ C^{\alpha^{\prime}} y^{\beta^{\prime}} z^{\gamma^{\prime}}$ if $C^{\alpha}>C^{\alpha^{\prime}}$ in the purely lexicographic order, or if $C^{\alpha}=C^{\alpha^{\prime}}$ and $y^{\beta}>y^{\beta^{\prime}}$ in the degree lexicographic order, or if $C^{\alpha}=C^{\alpha^{\prime}}$ and $y^{\beta}=y^{\beta^{\prime}}$ and $z^{\gamma}>z^{\gamma^{\prime}}$ in the purely lexicographic order. The resulting Gröbner basis will contain the desired syzygies.

To compute syzygies relating one secondary invariant $\phi_{i}$ to the primary invariants, essentially the same procedure is employed. The Gröbner basis of $F=\left\{\theta_{1}-\right.$ $\left.y_{1}, \ldots, \theta_{6}-y_{6}, \phi_{i}-z\right\}$ is computed in the variables $C_{1}>\cdots>C_{6}>y_{1}>\cdots>y_{6}>z$ with an order that eliminates the variables $C$. Good success was found using a matrix order (e.g., [9]) that first selects for graded degree (using the degrees in $C$ as weights), then selects for degree in the variables $C$, and then enforces reverse lexicographical ordering on the $C$ and $y$ blocks. As suggested by Bayer and Stillman [2], the reverse lexicographical refinement was substantially more efficient than purely lexicographical order.

The construction of Gröbner bases is given by a simple algorithm by Buchberger [5], but the simplicity of the algorithm belies the complexity of the computational

\footnotetext{
${ }^{1}$ Note that the Reynolds operator does depend on the "setting," or orientation, of the symmetry axes given in Table 3.1. To this point in the manuscript, only the setting-independent eigenvalues of the operations have been used.
} 
task. The maximum degree computed in a Gröbner basis may be as large as doubly exponential in the number of variables used [13]; and integer or rational coefficients have been reported to contain as many as $\mathcal{O}\left(10^{5}\right)$ significant decimal figures with basis functions containing $\mathcal{O}(1)$ coefficients [1]. Thus, poor algorithmic choices (and there are many choices one is free to make) render even simple basis calculations impossible. To control these issues directly, implementations of Buchberger's algorithm and the $F_{4}$ variant of this algorithm by Faugére [7] were constructed in $\mathrm{C}++$ using GMP $[12]$ to represent and manipulate arbitrary precision integers. Superfluous pairs were eliminated using the method of Gebauer and Möller [10], and selection strategies used the "sugar" phantom degree order method of [11]. The $F_{4}$ algorithm has been reported to be on the order of 10 times faster than the equivalent Buchberger method. Our implementation of $F_{4}$ modifies the selection criterion as follows. Let $\operatorname{deg}_{W}$ be a $W$-graded degree, chosen so all polynomials are $W$-homogeneous (e.g, weights $w_{i}$ correspond to the degree of a variable when expressed in the common basis of $C$ elements). An $F_{4}$ row echelon calculation containing polynomials of different $\operatorname{deg}_{W}$ may be immediately block diagonalized according to $\operatorname{deg}_{W}$. Including polynomials of different $\operatorname{deg}_{W}$ in a row echelon calculation does not affect the correctness of the method, but in practice it is found that selecting only those pairs whose $d e g_{W}$ are equal and as small as possible improves efficiency.

The results of these algorithms applied to the 11 Laue groups are presented below. The following subsections present the computed invariant bases, with elements distinguished as being primary or secondary invariants. In all cases the minimal factorizations displayed in Table 4.2 are realized. In the appendix, a complete set of rewriting syzygies is presented. Application of these equations may transform any polynomial $P(\{\theta\},\{\phi\})$ into the minimal form given by (4.5). These are offered in proof of the simplification implied by the Molien factorizations. Also presented in the appendix is a representative example of an algebraic dependence syzygy, a polynomial of the form $P\left(\phi_{\alpha}, \theta_{1}, \ldots, \theta_{6}\right)$ which demonstrates the algebraic dependence of the secondary invariants. Several such algebraic dependence syzygies also appear in the set of rewriting syzygies. Note that the computation of these algebraic dependence syzygies is difficult, and several such syzygies have thus far defied computation. With the algorithms used, the relevant Gröbner basis calculation may consume all available core memory $(8 \mathrm{~Gb})$ in the span of a few days.

5.1. Triclinic groups $C_{1}$ and $C_{\boldsymbol{i}}$. The group $C_{1}$ contains no symmetry operations aside from the identity $E$. The group $C_{i}$ contains only the identity and a center of inversion. With respect to the action of these groups on the Cauchy tensor components, the groups are therefore identical. Since no Cauchy tensor components are mixed by the action of these groups, there are no nontrivial Reynolds projections. The basis for these groups consists of the Cauchy tensor components, all primary invariants.

$$
\begin{aligned}
& \theta_{1}=C_{6}, \\
& \theta_{2}=C_{5}, \\
& \theta_{3}=C_{4}, \\
& \theta_{4}=C_{3}, \\
& \theta_{5}=C_{2}, \\
& \theta_{6}=C_{1} .
\end{aligned}
$$


5.2. Monoclinic groups $C_{s}, C_{2}, C_{2 h}$. A single secondary invariant exists for this group. An invariant basis is

$$
\begin{aligned}
\theta_{1} & =C_{4}, \\
\theta_{2} & =C_{3}, \\
\theta_{3} & =C_{2}, \\
\theta_{4} & =C_{1}, \\
\theta_{5} & =C_{6}^{2}, \\
\theta_{6} & =C_{5}^{2}, \\
\phi_{1} & =C_{5} C_{6} .
\end{aligned}
$$

5.3. Orthorhombic groups $C_{2 v}, D_{2}, D_{2 h}$. A single secondary invariant of degree 3 exists:

$$
\begin{aligned}
\theta_{1} & =C_{3}, \\
\theta_{2} & =C_{2}, \\
\theta_{3} & =C_{1}, \\
\theta_{4} & =C_{6}^{2}, \\
\theta_{5} & =C_{5}^{2}, \\
\theta_{6} & =C_{4}^{2}, \\
\phi_{1} & =C_{4} C_{5} C_{6} .
\end{aligned}
$$

5.4. Tetragonal groups $S_{4}, C_{4}, C_{4 h}$. An invariant basis obeying the Molien factorization of Table 4.2 is

$$
\begin{aligned}
& \theta_{1}=C_{3}, \\
& \theta_{2}=C_{1}+C_{2}, \\
& \theta_{3}=C_{6}^{2}, \\
& \theta_{4}=C_{4}^{2}+C_{5}^{2}, \\
& \theta_{5}=C_{1}^{2}+C_{2}^{2}, \\
& \theta_{6}=C_{4}^{4}+C_{5}^{4}, \\
& \phi_{1}=\left(C_{1}-C_{2}\right) C_{6}, \\
& \phi_{2}=\left(C_{4}^{2}-C_{5}^{2}\right) C_{6}, \\
& \phi_{3}=C_{4} C_{5} C_{6}, \\
& \phi_{4}=C_{1} C_{4}^{2}+C_{2} C_{5}^{2}, \\
& \phi_{5}=\left(C_{1}-C_{2}\right) C_{4} C_{5}, \\
& \phi_{6}=C_{4} C_{5}\left(C_{4}^{2}-C_{5}^{2}\right), \\
& \phi_{7}=\phi_{3} \phi_{4} .
\end{aligned}
$$

5.5. Tetragonal groups $D_{2 d}, C_{4 v}, D_{4}, D_{4 h}$. The invariant relations for these groups are also relatively simple:

$$
\begin{aligned}
& \theta_{1}=C_{3}, \\
& \theta_{2}=C_{1}+C_{2}, \\
& \theta_{3}=C_{6}^{2},
\end{aligned}
$$




$$
\begin{aligned}
\theta_{4} & =C_{4}^{2}+C_{5}^{2}, \\
\theta_{5} & =C_{1}^{2}+C_{2}^{2}, \\
\theta_{6} & =C_{4}^{4}+C_{5}^{4}, \\
\phi_{1} & =C_{4} C_{5} C_{6}, \\
\phi_{2} & =C_{1} C_{4}^{2}+C_{2} C_{5}^{2}, \\
\phi_{3} & =\phi_{1} \phi_{2} .
\end{aligned}
$$

5.6. Cubic groups $\boldsymbol{T}, \boldsymbol{T}_{\boldsymbol{h}}$ and groups $\boldsymbol{T}_{\boldsymbol{d}}, \boldsymbol{O}, \boldsymbol{O}_{\boldsymbol{h}}$. Group $T_{d}$ is subset of group $T$; they share the same primary invariants and several secondary invariants. Those secondary invariants found in group $T$ but absent from $T_{d}$ are denoted by an asterisk:

$$
\begin{aligned}
\theta_{1} & =C_{1}+C_{2}+C_{3}, \\
\theta_{2} & =C_{4}^{2}+C_{5}^{2}+C_{6}^{2}, \\
\theta_{3} & =C_{1}^{2}+C_{2}^{2}+C_{3}^{2}, \\
\theta_{4} & =C_{4} C_{5} C_{6}, \\
\theta_{5} & =C_{1}^{3}+C_{2}^{3}+C_{3}^{3}, \\
\theta_{6} & =C_{4}^{4}+C_{5}^{4}+C_{6}^{4}, \\
\phi_{1} & =C_{1} C_{4}^{2}+C_{2} C_{5}^{2}+C_{3} C_{6}^{2}, \\
\phi_{2} & =\phi_{1}^{2}, \\
\phi_{3} & =\phi_{1}^{3}, \\
(*) \quad \phi_{4} & =C_{1} C_{6}^{2}+C_{2} C_{4}^{2}+C_{3} C_{5}^{2}, \\
(*) \quad \phi_{5} & =\phi_{4}^{2}, \\
(*) \quad \phi_{6} & =C_{1}^{2} C_{3}+C_{1} C_{2}^{2}+C_{2} C_{3}^{2}, \\
\phi_{7} & =C_{1}^{2} C_{4}^{2}+C_{2}^{2} C_{5}^{2}+C_{3}^{2} C_{6}^{2}, \\
(*) \quad \phi_{8} & =C_{1}^{2} C_{6}^{2}+C_{2}^{2} C_{4}^{2}+C_{3}^{2} C_{5}^{2}, \\
\phi_{9} & =C_{1} C_{4}^{4}+C_{2} C_{5}^{4}+C_{3} C_{6}^{4}, \\
(*) \quad \phi_{10} & =C_{1} C_{4}^{2} C_{6}^{2}+C_{2} C_{4}^{2} C_{5}^{2}+C_{3} C_{5}^{2} C_{6}^{2}, \\
(*) \quad \phi_{11} & =C_{4}^{4} C_{6}^{2}+C_{4}^{2} C_{5}^{4}+C_{5}^{2} C_{6}^{4} .
\end{aligned}
$$

It is interesting to note that the groups $T$ and $T_{d}$ share the same primary invariants. Consider (A.62), an algebraic dependence syzygy for $\phi_{4}$, which occurs in $T$ but not in $T_{d}$. The coefficients of $\phi_{4}^{m}, m \in(0,6)$, in (A.62) are expressed in terms of $\theta$, and therefore the coefficients are invariant with respect to both $T$ and $T_{d}$. However, the roots of this syzygy are not invariant. In $T$ the roots $\phi$ of (A.62) describe an orbit of size 6 under the action of the reflection symmetry operations found in $T$ but not in $T_{d}$.

5.7. Trigonal groups $C_{3}, S_{6}$. An invariant basis is

$$
\begin{aligned}
& \theta_{1}=C_{3}, \\
& \theta_{2}=C_{1}+C_{2}, \\
& \theta_{3}=\left(C_{1}-C_{2}\right)^{2}+C_{6}^{2}, \\
& \theta_{4}=C_{4}^{2}+C_{5}^{2}, \\
& \theta_{5}=C_{6}^{3}-3 C_{6}\left(C_{1}-C_{2}\right)^{2},
\end{aligned}
$$


5.8. Trigonal groups $C_{3 v}, D_{3}, D_{3 d}$. An invariant basis is

$$
\begin{aligned}
\theta_{6} & =C_{5}\left(C_{5}^{2}-3 C_{4}^{2}\right), \\
\phi_{1} & =C_{4}\left(C_{1}-C_{2}\right)+C_{5} C_{6}, \\
\phi_{2} & =\phi_{1}^{2}, \\
\phi_{3} & =\phi_{1}^{3}, \\
\phi_{4} & =C_{5}\left(C_{1}-C_{2}\right)-C_{4} C_{6}, \\
\phi_{5} & =C_{5}\left(C_{1}-C_{2}\right)^{2}+2 C_{4} C_{6}\left(C_{1}-C_{2}\right)-C_{5} C_{6}^{2}, \\
\phi_{6} & =2 C_{4} C_{5}\left(C_{1}-C_{2}\right)+C_{6}\left(C_{4}^{2}-C_{5}^{2}\right), \\
\phi_{7} & =C_{4}\left(C_{1}-C_{2}\right)^{2}-2 C_{5} C_{6}\left(C_{1}-C_{2}\right)-C_{4} C_{6}^{2}, \\
\phi_{8} & =\left(C_{1}-C_{2}\right)\left(C_{4}^{2}-C_{5}^{2}\right)-2 C_{4} C_{5} C_{6}, \\
\phi_{9} & =C_{4}\left(C_{4}^{2}-3 C_{5}^{2}\right), \\
\phi_{10} & =\left(C_{1}-C_{2}\right)^{2}\left(3 C_{1}+C_{2}\right)-C_{6}^{2}\left(C_{1}-5 C_{2}\right), \\
\phi_{11} & =\phi_{1} \phi_{4} .
\end{aligned}
$$

$$
\begin{aligned}
& \theta_{1}=C_{3}, \\
& \theta_{2}=C_{1}+C_{2}, \\
& \theta_{3}=\left(C_{1}-C_{2}\right)^{2}+C_{6}^{2}, \\
& \theta_{4}=C_{4}^{2}+C_{5}^{2}, \\
& \theta_{5}=C_{4}\left(C_{4}^{2}-3 C_{5}^{2}\right), \\
& \theta_{6}=\left(C_{1}-C_{2}\right)^{2}\left(3 C_{1}+C_{2}\right)-C_{6}^{2}\left(C_{1}-5 C_{2}\right), \\
& \phi_{1}=C_{4}\left(C_{1}-C_{2}\right)+C_{5} C_{6}, \\
& \phi_{2}=\phi_{1}^{2}, \\
& \phi_{3}=\phi_{1}^{3}, \\
& \phi_{4}=C_{4}\left(C_{1}-C_{2}\right)^{2}-2 C_{5} C_{6}\left(C_{1}-C_{2}\right)-C_{4} C_{6}^{2}, \\
& \phi_{5}=\left(C_{1}-C_{2}\right)\left(C_{4}^{2}-C_{5}^{2}\right)-2 C_{4} C_{5} C_{6} .
\end{aligned}
$$

5.9. Hexagonal groups $C_{3 h}, C_{6}, C_{6 h}$. An invariant basis is

$$
\begin{aligned}
& (5.88) \quad \theta_{1}=C_{3} \\
& (5.89) \quad \theta_{2}=C_{1}+C_{2} \\
& (5.90) \quad \theta_{3}=\left(C_{1}-C_{2}\right)^{2}+C_{6}^{2}, \\
& (5.91) \quad \theta_{4}=C_{4}^{2}+C_{5}^{2} \\
& (5.92) \quad \theta_{5}=C_{6}^{3}-3 C_{6}\left(C_{1}-C_{2}\right)^{2} \\
& (5.93) \quad \theta_{6}=9 C_{4}^{6}+45 C_{4}^{4} C_{5}^{2}+15 C_{4}^{2} C_{5}^{4}+11 C_{5}^{6}, \\
& (5.94) \quad \phi_{1}=2 C_{4} C_{5}\left(C_{1}-C_{2}\right)+C_{6}\left(C_{4}^{2}-C_{5}^{2}\right), \\
& (5.95) \quad \phi_{2}=\phi_{1}^{2} \\
& (5.96) \quad \phi_{3}=\phi_{1}^{3} \\
& (5.97) \quad \phi_{4}=\left(C_{1}-C_{2}\right)\left(C_{4}^{2}-C_{5}^{2}\right)-2 C_{4} C_{5} C_{6}, \\
& (5.98) \quad \phi_{5}=\left(C_{1}-C_{2}\right)^{2}\left(3 C_{1}+C_{2}\right)-C_{6}^{2}\left(C_{1}-5 C_{2}\right), \\
& (5.99) \quad \phi_{6}=\left(3 C_{4}^{2}+C_{5}^{2}\right)\left(C_{1}-C_{2}\right)^{2}+4 C_{4} C_{5} C_{6}\left(C_{1}-C_{2}\right)+C_{6}^{2}\left(C_{4}^{2}+3 C_{5}^{2}\right), \\
& (5.100) \quad \phi_{7}=\left(C_{1} C_{5}-C_{2} C_{5}-C_{4} C_{6}\right)\left(C_{1} C_{4}-C_{2} C_{4}+C_{5} C_{6}\right),
\end{aligned}
$$




$$
\begin{aligned}
\phi_{8}= & 4 C_{4} C_{5}\left(C_{1}-C_{2}\right)\left(3 C_{4}^{2}+C_{5}^{2}\right)+C_{6}\left(3 C_{4}^{4}+6 C_{4}^{2} C_{5}^{2}-5 C_{5}^{4}\right) \\
\phi_{9}= & 8 C_{4} C_{5}^{3} C_{6}-\left(C_{1}-C_{2}\right)\left(3 C_{4}^{4}-6 C_{4}^{2} C_{5}^{2}-C_{5}^{4}\right) \\
\phi_{10}= & 4 C_{4} C_{5}^{3}\left(C_{1}-C_{2}\right)^{2}-4 C_{4} C_{5}^{3} C_{6}^{2}-\left(C_{1}-C_{2}\right) \\
& \times\left(3 C_{4}^{4} C_{6}-6 C_{4}^{2} C_{5}^{2} C_{6}-C_{5}^{4} C_{6}\right) \\
\phi_{11}= & -C_{4} C_{5}\left(C_{4}^{2}-3 C_{5}^{2}\right)\left(3 C_{4}^{2}-C_{5}^{2}\right)
\end{aligned}
$$

5.10. Hexagonal groups $D_{3 h}, C_{6 v}, D_{6}, D_{6 h}$. An invariant basis for these groups is

$$
\begin{aligned}
\theta_{1}= & C_{3}, \\
\theta_{2}= & C_{1}+C_{2}, \\
\theta_{3}= & \left(C_{1}-C_{2}\right)^{2}+C_{6}^{2}, \\
\theta_{4}= & C_{4}^{2}+C_{5}^{2}, \\
\theta_{5}= & \left(C_{1}-C_{2}\right)^{2}\left(3 C_{1}+C_{2}\right)-C_{6}^{2}\left(C_{1}-5 C_{2}\right), \\
\theta_{6}= & 9 C_{4}^{6}+45 C_{4}^{4} C_{5}^{2}+15 C_{4}^{2} C_{5}^{4}+11 C_{5}^{6}, \\
\phi_{1}= & \left(C_{1}-C_{2}\right)\left(C_{4}^{2}-C_{5}^{2}\right)-2 C_{4} C_{5} C_{6}, \\
\phi_{2}= & \phi_{1}^{2}, \\
\phi_{3}= & \phi_{1}^{3}, \\
\phi_{4}= & \left(C_{1}-C_{2}\right)^{2}\left(3 C_{4}^{2}+C_{5}^{2}\right)+4 C_{4} C_{5} C_{6} \\
& \times\left(C_{1}-C_{2}\right)+C_{6}^{2}\left(C_{4}^{2}+3 C_{5}^{2}\right), \\
\phi_{5}= & 8 C_{4} C_{5}^{3} C_{6}-\left(C_{1}-C_{2}\right)\left(3 C_{4}^{4}-6 C_{4}^{2} C_{5}^{2}-C_{5}^{4}\right) .
\end{aligned}
$$

6. Conclusions. The invariant bases presented above agree with those presented by Smith and Rivlin [20] and are identical in the sense that they generate the same ideal. In many cases the particular form of the invariants differs. This has no significance and is merely an artifact of the particular methods used. For example, in the group $T$ the invariants $K$ presented by Smith and Rivlin are related to the invariants $\theta$ and $\phi$ in (5.43)-(5.59) via $\theta_{1}=K_{1}, \theta_{2}=K_{4}, \theta_{3}=K_{1}^{2}-2 K_{2}$, $\theta_{4}=K_{0}, \theta_{5}=K_{1}^{3}-3 K_{1} K_{2}+3 K_{3}, \theta_{6}=K_{4}^{2}-2 K_{5}, \phi_{1}=K_{1} K_{4}-K_{7}-K_{8}$, $\phi_{4}=K_{8}, \phi_{6}=K_{1} K_{2}-K_{9}-3 K_{3}, \phi_{7}=K_{1}^{2} K_{4}-K_{1} K_{7}-K_{1} K_{8}+K_{12}-K_{2} K_{4}$, $\phi_{8}=K_{1} K_{8}-K_{2} K_{4}+K_{13}, \phi_{9}=K_{1} K_{4}^{2}-K_{4} K_{8}-K_{4} K_{7}-K_{1} K_{5}+K_{11}, \phi_{10}=K_{14}$, and $\phi_{11}=K_{4} K_{5}-K_{10}-3 K_{6}$ (with $K_{0}=\sqrt{K_{6}}=C_{4} C_{5} C_{6}$ ). By writing the $K$ 's in terms of $\theta$ 's and $\phi$ 's, it is apparent on inspection that $K_{1}, K_{4}, K_{2}, K_{0}, K_{3}$, and $K_{5}$ form an HSOP and a set of primary invariants of minimal degree indicated in Table 4.2. Likewise, $K_{7}, K_{8}, K_{9}, K_{12}, K_{13}, K_{11}, K_{14}$, and $K_{10}$ are valid secondary invariants. To make a complete set of secondary invariants, one could include both $K_{7}^{2}$ and $K_{8}^{2}$ of degree 6 and one of $K_{7}^{3}$ or $K_{8}^{3}$ of degree 9 .

The following truncated series displays the difference between the Hilbert series implied by the Smith and Rivlin integrity bases and the invariants deduced above in their Molien form. The Smith and Rivlin results differ beginning with fourth-degree (in $C$ ) polynomials.

(6.2) $C_{2 v}: z^{6}+3 z^{7}+9 z^{8}+20 z^{9}+42 z^{10}+78 z^{11}+139 z^{12}+\cdots$,

(6.3) $S_{4}: \quad 2 z^{4}+8 z^{5}+32 z^{6}+80 z^{7}+194 z^{8}+404 z^{9}+808 z^{10}+1488 z^{11}+2663 z^{12}+\cdots$,

(6.4) $D_{2 d}: 2 z^{6}+4 z^{7}+12 z^{8}+24 z^{9}+50 z^{10}+88 z^{11}+157 z^{12}+\cdots$, 
$(6.5)$

$$
\begin{array}{lrl}
(6.5) & T: & 4 z^{6}+10 z^{7}+27 z^{8}+63 z^{9}+126 z^{10}+239 z^{11}+439 z^{12}+\cdots, \\
(6.6) & T_{d}: & z^{7}+3 z^{8}+6 z^{9}+14 z^{10}+26 z^{11}+47 z^{12}+\cdots, \\
(6.7) & C_{3}: & z^{4}+14 z^{5}+53 z^{6}+136 z^{7}+341 z^{8}+750 z^{9}+1485 z^{10}+2856 z^{11}+5206 z^{12}+\cdots, \\
(6.8) & C_{3 v}: & 2 z^{5}+7 z^{6}+18 z^{7}+43 z^{8}+90 z^{9}+170 z^{10}+308 z^{11}+528 z^{12}+\cdots, \\
(6.9) & C_{3 h}: & 4 z^{6}+14 z^{7}+41 z^{8}+100 z^{9}+212 z^{10}+414 z^{11}+767 z^{12}+\cdots, \\
(6.10) & D_{3 h}: & z^{7}+4 z^{8}+10 z^{9}+23 z^{10}+45 z^{11}+83 z^{12}+\cdots
\end{array}
$$

\section{Appendix. Syzygies.}

The invariant bases for the triclinic groups $C_{1}$ and $C_{i}$ contain no secondary invariants and hence no syzygies.

A.1. Monoclinic groups $C_{s}, C_{2}, C_{2 h}$. This syzygy is a rewriting expression and also displays the algebraic dependence of the secondary invariant upon the HSOP:

$$
\phi_{1}^{2}=\theta_{5} \theta_{6} .
$$

A.2. Orthorhombic groups $C_{2 v}, D_{2}, D_{2 h}$. An obvious syzygy exists:

$$
\phi_{1}^{2}=\theta_{4} \theta_{5} \theta_{6} .
$$

This is a rewriting expression and displays the algebraic dependence.

A.3. Tetragonal groups $S_{4}, C_{4}, C_{4 h}$. Again, the algebraic dependence syzygies are included in the set of rewriting syzygies. Rewriting syzygies for $\phi_{7} \phi_{\alpha}$ are omitted since they may be simply constructed by rewriting $\phi_{3}\left(\phi_{4} \phi_{\alpha}\right)$ or $\phi_{4}\left(\phi_{3} \phi_{\alpha}\right)$.

$$
\begin{aligned}
\phi_{1}^{2} & =-\theta_{3}\left[\theta_{2}^{2}-2 \theta_{5}\right], \\
\phi_{1} \phi_{2} & =-\theta_{2} \theta_{3} \theta_{4}+2 \theta_{3} \phi_{4}, \\
\phi_{1} \phi_{3} & =\theta_{3} \phi_{5}, \\
\phi_{1} \phi_{4} & =\frac{1}{2} \theta_{2} \theta_{4} \phi_{1}-\frac{1}{2}\left[\theta_{2}^{2}-2 \theta_{5}\right] \phi_{2}, \\
\phi_{1} \phi_{5} & =-\left[\theta_{2}^{2}-2 \theta_{5}\right] \phi_{3}, \\
\phi_{1} \phi_{6} & =-\theta_{2} \theta_{4} \phi_{3}+2 \phi_{7}, \\
\phi_{2}^{2} & =-\theta_{3}\left[\theta_{4}^{2}-2 \theta_{6}\right], \\
\phi_{2} \phi_{3} & =\theta_{3} \phi_{6}, \\
\phi_{2} \phi_{4} & =-\frac{1}{2}\left[\theta_{4}^{2}-2 \theta_{6}\right] \phi_{1}+\frac{1}{2} \theta_{2} \theta_{4} \phi_{2}, \\
\phi_{2} \phi_{5} & =\phi_{1} \phi_{6} \\
& =-\theta_{2} \theta_{4} \phi_{3}+2 \phi_{7}, \\
\phi_{2} \phi_{6} & =-\left[\theta_{4}^{2}-2 \theta_{6}\right] \phi_{3}, \\
\phi_{3}^{2} & =\frac{1}{2} \theta_{3}\left[\theta_{4}^{2}-\theta_{6}\right], \\
\phi_{3} \phi_{4} & =\phi_{7}, \\
\phi_{3} \phi_{5} & =\frac{1}{2}\left[\theta_{4}^{2}-\theta_{6}\right] \phi_{1}, \\
\phi_{3} \phi_{6} & =\frac{1}{2}\left[\theta_{4}^{2}-\theta_{6}\right] \phi_{2}, \\
\phi_{4}^{2} & =-\frac{1}{2}\left[\theta_{2}^{2} \theta_{6}+\theta_{4}^{2} \theta_{5}-2 \theta_{5} \theta_{6}\right]+\theta_{2} \theta_{4} \phi_{4},
\end{aligned}
$$




$$
\begin{aligned}
\phi_{4} \phi_{5} & =\frac{1}{2} \theta_{2} \theta_{4} \phi_{5}-\frac{1}{2}\left[\theta_{2}^{2}-2 \theta_{5}\right] \phi_{6}, \\
\phi_{4} \phi_{6} & =-\frac{1}{2}\left[\theta_{4}^{2}-2 \theta_{6}\right] \phi_{5}+\frac{1}{2} \theta_{2} \theta_{4} \phi_{6}, \\
\phi_{5}^{2} & =-\frac{1}{2}\left[\theta_{2}^{2} \theta_{4}^{2}-\theta_{2}^{2} \theta_{6}-2 \theta_{4}^{2} \theta_{5}+2 \theta_{5} \theta_{6}\right], \\
\phi_{5} \phi_{6} & =-\frac{1}{2} \theta_{2} \theta_{4}\left[\theta_{4}^{2}-\theta_{6}\right]+\left[\theta_{4}^{2}-\theta_{6}\right] \phi_{4}, \\
\phi_{6}^{2} & =-\frac{1}{2}\left[\theta_{4}^{4}-3 \theta_{4}^{2} \theta_{6}+2 \theta_{6}^{2}\right] .
\end{aligned}
$$

A.4. Tetragonal groups $D_{2 d}, C_{4 v}, D_{4}, D_{4 h}$. Algebraic dependence syzygies coincide with the rewriting syzygies for these groups:

$$
\begin{aligned}
\phi_{1}^{2} & =\frac{1}{2} \theta_{3}\left[\theta_{4}^{2}-\theta_{6}\right], \\
\phi_{2}^{2} & =-\frac{1}{2}\left[\theta_{2}^{2} \theta_{6}+\theta_{4}^{2} \theta_{5}-2 \theta_{5} \theta_{6}\right]+\theta_{2} \theta_{4} \phi_{2} .
\end{aligned}
$$

A.5. Cubic groups $T, T_{h}$ and groups $T_{d}, O, O_{h}$. The rewriting syzygies for this group are complicated and do not contain all algebraic dependence syzygies. Note that the rewriting syzygies for invariants of group $T_{d}$ are expressed in terms of primaries $\theta$ and only those secondary invariants of group $T_{d}$.

$$
\begin{aligned}
& \phi_{1}^{4}=\frac{1}{36}\left[2 \theta_{1}^{4} \theta_{2}^{4}-7 \theta_{1}^{4} \theta_{2}^{2} \theta_{6}-12 \theta_{1}^{4} \theta_{2} \theta_{4}^{2}+3 \theta_{1}^{4} \theta_{6}^{2}-10 \theta_{1}^{2} \theta_{2}^{4} \theta_{3}\right. \\
& +28 \theta_{1}^{2} \theta_{2}^{2} \theta_{3} \theta_{6}+54 \theta_{1}^{2} \theta_{2} \theta_{3} \theta_{4}^{2}-12 \theta_{1}^{2} \theta_{3} \theta_{6}^{2}+14 \theta_{1} \theta_{2}^{4} \theta_{5} \\
& -36 \theta_{1} \theta_{2}^{2} \theta_{5} \theta_{6}-108 \theta_{1} \theta_{2} \theta_{4}^{2} \theta_{5}+18 \theta_{1} \theta_{5} \theta_{6}^{2}-6 \theta_{2}^{4} \theta_{3}^{2}+15 \theta_{2}^{2} \theta_{3}^{2} \theta_{6} \\
& \left.+54 \theta_{2} \theta_{3}^{2} \theta_{4}^{2}-9 \theta_{3}^{2} \theta_{6}^{2}\right]-\frac{1}{18}\left[2 \theta_{1}^{3} \theta_{2}^{3}-12 \theta_{1}^{3} \theta_{2} \theta_{6}-18 \theta_{1}^{3} \theta_{4}^{2}\right. \\
& -21 \theta_{1} \theta_{2}^{3} \theta_{3}+51 \theta_{1} \theta_{2} \theta_{3} \theta_{6}+108 \theta_{1} \theta_{3} \theta_{4}^{2}+21 \theta_{2}^{3} \theta_{5}-45 \theta_{2} \theta_{5} \theta_{6} \\
& \left.-162 \theta_{4}^{2} \theta_{5}\right] \phi_{1}-\frac{1}{36}\left[19 \theta_{1}^{2} \theta_{2}^{2}+15 \theta_{1}^{2} \theta_{6}+15 \theta_{2}^{2} \theta_{3}-45 \theta_{3} \theta_{6}\right] \phi_{2} \\
& +\frac{4}{3} \theta_{1} \theta_{2} \phi_{3}-\frac{1}{36}\left[\theta_{1}^{2}-3 \theta_{3}\right]\left[5 \theta_{2}^{3}-9 \theta_{2} \theta_{6}-54 \theta_{4}^{2}\right] \phi_{7} \\
& +\frac{1}{18}\left[2 \theta_{1}^{3}-9 \theta_{1} \theta_{3}+9 \theta_{5}\right]\left[\theta_{2}^{2}-3 \theta_{6}\right] \phi_{9}, \\
& \text { (A.27) } \phi_{1} \phi_{4}=-\frac{1}{4}\left[\theta_{1}^{2} \theta_{2}^{2}+\theta_{1}^{2} \theta_{6}+\theta_{2}^{2} \theta_{3}-3 \theta_{3} \theta_{6}\right]+\theta_{1} \theta_{2} \phi_{1}-\phi_{2}+\theta_{1} \theta_{2} \phi_{4}-\phi_{5} \text {, } \\
& \phi_{1} \phi_{6}=-\frac{1}{6} \theta_{2}\left[\theta_{1}^{2} \theta_{3}+3 \theta_{3}^{2}-4 \theta_{1} \theta_{5}\right]+\frac{1}{3}\left[2 \theta_{1} \theta_{3}-3 \theta_{5}\right] \phi_{1}+\frac{1}{3}\left[\theta_{1} \theta_{3}-3 \theta_{5}\right] \phi_{4} \\
& +\frac{1}{3} \theta_{1} \theta_{2} \phi_{6}-\frac{1}{6}\left[\theta_{1}^{2}-3 \theta_{3}\right] \phi_{7}-\frac{1}{3}\left[\theta_{1}^{2}-3 \theta_{3}\right] \phi_{8}, \\
& \phi_{1} \phi_{7}=\frac{1}{6}\left[\theta_{1}^{3} \theta_{6}+\theta_{1} \theta_{2}^{2} \theta_{3}-4 \theta_{1} \theta_{3} \theta_{6}-\theta_{2}^{2} \theta_{5}+3 \theta_{5} \theta_{6}\right]-\frac{1}{3} \theta_{1}^{2} \theta_{2} \phi_{1} \\
& +\frac{2}{3} \theta_{1} \phi_{2}+\frac{1}{3} \theta_{1} \theta_{2} \phi_{7}-\frac{1}{6}\left[\theta_{1}^{2}-3 \theta_{3}\right] \phi_{9}, \\
& \phi_{1} \phi_{8}=-\frac{1}{12} \theta_{1}\left[\theta_{1}^{2} \theta_{2}^{2}+3 \theta_{1}^{2} \theta_{6}+3 \theta_{2}^{2} \theta_{3}-7 \theta_{3} \theta_{6}\right]+\frac{1}{6} \theta_{2}\left[3 \theta_{1}^{2}+\theta_{3}\right] \phi_{1} \\
& -\frac{2}{3} \theta_{1} \phi_{2}+\frac{1}{6} \theta_{2}\left[3 \theta_{1}^{2}-\theta_{3}\right] \phi_{4}-\frac{2}{3} \theta_{1} \phi_{5}-\frac{1}{6}\left[\theta_{2}^{2}-3 \theta_{6}\right] \phi_{6} \\
& +\frac{1}{3} \theta_{1} \theta_{2} \phi_{8}-\frac{1}{6}\left[\theta_{1}^{2}-3 \theta_{3}\right] \phi_{10}
\end{aligned}
$$




$$
\begin{aligned}
\phi_{1} \phi_{9}= & \frac{1}{12}\left[\theta_{1}^{2} \theta_{2}^{3}-\theta_{1}^{2} \theta_{2} \theta_{6}-6 \theta_{1}^{2} \theta_{4}^{2}-\theta_{2}^{3} \theta_{3}+\theta_{2} \theta_{3} \theta_{6}+18 \theta_{3} \theta_{4}^{2}\right] \\
& -\frac{1}{3} \theta_{1} \theta_{2}^{2} \phi_{1}+\frac{2}{3} \theta_{2} \phi_{2}-\frac{1}{6}\left[\theta_{2}^{2}-3 \theta_{6}\right] \phi_{7}+\frac{1}{3} \theta_{1} \theta_{2} \phi_{9},
\end{aligned}
$$

$$
\begin{aligned}
\phi_{1} \phi_{10}= & -\frac{1}{12} \theta_{2}\left[\theta_{1}^{2}+\theta_{3}\right]\left[\theta_{2}^{2}-\theta_{6}\right]-\frac{1}{12} \theta_{2}\left[\theta_{1}^{2} \theta_{2}^{2}-\theta_{1}^{2} \theta_{6}+\theta_{2}^{2} \theta_{3}-\theta_{3} \theta_{6}\right] \\
& +\frac{1}{6} \theta_{1}\left[\theta_{2}^{2}-\theta_{6}\right] \phi_{1}+\frac{1}{3} \theta_{1}\left[\theta_{2}^{2}-\theta_{6}\right] \phi_{4}-\frac{1}{3} \theta_{2} \phi_{5} \\
& -\frac{1}{6}\left[\theta_{2}^{2}-3 \theta_{6}\right] \phi_{8}+\frac{1}{3} \theta_{1} \theta_{2} \phi_{10}-\frac{1}{6}\left[\theta_{1}^{2}-3 \theta_{3}\right] \phi_{11},
\end{aligned}
$$

$$
\begin{aligned}
\phi_{1} \phi_{11}= & -\theta_{1} \theta_{2} \theta_{4}^{2}+\frac{1}{3} \theta_{2}\left[\theta_{2}^{2}-2 \theta_{6}\right] \phi_{1}-\frac{1}{6}\left[\theta_{2}^{3}-\theta_{2} \theta_{6}-18 \theta_{4}^{2}\right] \phi_{4} \\
& -\frac{1}{6}\left[\theta_{2}^{2}-3 \theta_{6}\right] \phi_{9}-\frac{1}{3}\left[\theta_{2}^{2}-3 \theta_{6}\right] \phi_{10}+\frac{1}{3} \theta_{1} \theta_{2} \phi_{11},
\end{aligned}
$$$$
\phi_{4}^{3}=\frac{1}{4}\left[\theta_{1}^{2} \theta_{2}^{2}+\theta_{1}^{2} \theta_{6}+\theta_{2}^{2} \theta_{3}-3 \theta_{3} \theta_{6}\right] \phi_{1}-\theta_{1} \theta_{2} \phi_{2}+\phi_{3}
$$$$
-\frac{1}{4}\left[\theta_{1}^{2} \theta_{2}^{2}+\theta_{1}^{2} \theta_{6}+\theta_{2}^{2} \theta_{3}-3 \theta_{3} \theta_{6}\right] \phi_{4}+\theta_{1} \theta_{2} \phi_{5},
$$$$
\phi_{4} \phi_{6}=-\frac{1}{6} \theta_{2}\left[\theta_{1}^{2} \theta_{3}-3 \theta_{3}^{2}+2 \theta_{1} \theta_{5}\right]-\frac{1}{3}\left[\theta_{1} \theta_{3}-3 \theta_{5}\right] \phi_{1}+\frac{1}{3} \theta_{1} \theta_{3} \phi_{4}
$$$$
+\frac{1}{3} \theta_{1} \theta_{2} \phi_{6}+\frac{1}{3}\left[\theta_{1}^{2}-3 \theta_{3}\right] \phi_{7}+\frac{1}{6}\left[\theta_{1}^{2}-3 \theta_{3}\right] \phi_{8},
$$$$
\phi_{4} \phi_{7}=-\frac{1}{12}\left[\theta_{1}^{3} \theta_{2}^{2}+3 \theta_{1}^{3} \theta_{6}+5 \theta_{1} \theta_{2}^{2} \theta_{3}-13 \theta_{1} \theta_{3} \theta_{6}-2 \theta_{2}^{2} \theta_{5}+6 \theta_{5} \theta_{6}\right]
$$

$$
\begin{aligned}
& +\frac{1}{6} \theta_{2}\left[3 \theta_{1}^{2}-\theta_{3}\right] \phi_{1}-\frac{2}{3} \theta_{1} \phi_{2}+\frac{1}{6} \theta_{2}\left[3 \theta_{1}^{2}+\theta_{3}\right] \phi_{4}-\frac{2}{3} \theta_{1} \phi_{5} \\
& +\frac{1}{6}\left[\theta_{2}^{2}-3 \theta_{6}\right] \phi_{6}+\frac{1}{3} \theta_{1} \theta_{2} \phi_{7}-\frac{1}{6}\left[\theta_{1}^{2}-3 \theta_{3}\right] \phi_{10},
\end{aligned}
$$

$$
\phi_{4} \phi_{8}=\frac{1}{12}\left[\theta_{1}^{3} \theta_{2}^{2}+\theta_{1}^{3} \theta_{6}-\theta_{1} \theta_{2}^{2} \theta_{3}-5 \theta_{1} \theta_{3} \theta_{6}-2 \theta_{2}^{2} \theta_{5}+6 \theta_{5} \theta_{6}\right]
$$$$
-\frac{1}{6} \theta_{2}\left[\theta_{1}^{2}-3 \theta_{3}\right] \phi_{1}-\frac{1}{2} \theta_{2}\left[\theta_{1}^{2}-\theta_{3}\right] \phi_{4}+\frac{2}{3} \theta_{1} \phi_{5}
$$

$$
\begin{aligned}
& +\frac{1}{3} \theta_{1} \theta_{2} \phi_{8}+\frac{1}{6}\left[\theta_{1}^{2}-3 \theta_{3}\right] \phi_{9}+\frac{1}{6}\left[\theta_{1}^{2}-3 \theta_{3}\right] \phi_{10}, \\
\phi_{4} \phi_{9}= & -\frac{1}{6} \theta_{2}\left[3 \theta_{1}^{2} \theta_{6}+2 \theta_{2}^{2} \theta_{3}-5 \theta_{3} \theta_{6}\right]+\frac{1}{3} \theta_{1}\left[\theta_{2}^{2}+\theta_{6}\right] \phi_{1}-\frac{2}{3} \theta_{2} \phi_{2} \\
& +\frac{1}{3} \theta_{1}\left[\theta_{2}^{2}+2 \theta_{6}\right] \phi_{4}-\frac{2}{3} \theta_{2} \phi_{5}+\frac{1}{6}\left[\theta_{2}^{2}-3 \theta_{6}\right] \phi_{7} \\
& +\frac{1}{6}\left[\theta_{2}^{2}-3 \theta_{6}\right] \phi_{8}+\frac{1}{3} \theta_{1} \theta_{2} \phi_{9}-\frac{1}{6}\left[\theta_{1}^{2}-3 \theta_{3}\right] \phi_{11}, \\
\phi_{4} \phi_{10}= & -\frac{1}{6}\left[\theta_{1}^{2} \theta_{2}^{3}-3 \theta_{1}^{2} \theta_{4}^{2}-\theta_{1}^{2} \theta_{2} \theta_{6}-\theta_{2}^{3} \theta_{3}+\theta_{2} \theta_{3} \theta_{6}+9 \theta_{3} \theta_{4}^{2}\right]
\end{aligned}
$$$$
+\frac{1}{3} \theta_{1}\left[\theta_{2}^{2}-\theta_{6}\right] \phi_{1}-\frac{1}{3} \theta_{2} \phi_{2}+\frac{1}{6} \theta_{1}\left[\theta_{2}^{2}-\theta_{6}\right] \phi_{4}-\frac{1}{6}\left[\theta_{2}^{2}-3 \theta_{6}\right] \phi_{7}
$$

$$
+\frac{1}{3} \theta_{1} \theta_{2} \phi_{10}+\frac{1}{6}\left[\theta_{1}^{2}-3 \theta_{3}\right] \phi_{11},
$$

$$
\phi_{4} \phi_{11}=-\frac{1}{4} \theta_{1}\left[\theta_{2}^{4}-2 \theta_{2}^{2} \theta_{6}-8 \theta_{2} \theta_{4}^{2}+\theta_{6}^{2}\right]+\frac{1}{3}\left[\theta_{2}^{3}-2 \theta_{2} \theta_{6}-9 \theta_{4}^{2}\right] \phi_{1}
$$

$$
\begin{aligned}
& +\frac{1}{3}\left[\theta_{2}^{3}-\theta_{2} \theta_{6}-9 \theta_{4}^{2}\right] \phi_{4}-\frac{1}{6}\left[\theta_{2}^{2}-3 \theta_{6}\right] \phi_{9} \\
& +\frac{1}{6}\left[\theta_{2}^{2}-3 \theta_{6}\right] \phi_{10}+\frac{1}{3} \theta_{1} \theta_{2} \phi_{11}, \\
\phi_{6}^{2}= & -\frac{1}{24}\left[\theta_{1}^{6}-9 \theta_{1}^{4} \theta_{3}+8 \theta_{1}^{3} \theta_{5}+27 \theta_{1}^{2} \theta_{3}^{2}-48 \theta_{1} \theta_{3} \theta_{5}-3 \theta_{3}^{3}+24 \theta_{5}^{2}\right] \\
& +\left[\theta_{1} \theta_{3}-\theta_{5}\right] \phi_{6},
\end{aligned}
$$


(A.42)

$$
\begin{aligned}
\phi_{6} \phi_{7}= & \frac{1}{12} \theta_{2}\left[\theta_{1}^{5}-6 \theta_{1}^{3} \theta_{3}+8 \theta_{1}^{2} \theta_{5}+\theta_{1} \theta_{3}^{2}-4 \theta_{3} \theta_{5}\right]-\frac{1}{12}\left[\theta_{1}^{4}-6 \theta_{1}^{2} \theta_{3}+\theta_{3}^{2}+8 \theta_{1} \theta_{5}\right] \phi_{1} \\
& -\frac{1}{6}\left[\theta_{1}^{4}-6 \theta_{1}^{2} \theta_{3}+8 \theta_{1} \theta_{5}+\theta_{3}^{2}\right] \phi_{4}+\frac{1}{3} \theta_{2} \theta_{3} \phi_{6} \\
& +\frac{1}{3} \theta_{1} \theta_{3} \phi_{7}-\frac{1}{3}\left[\theta_{1} \theta_{3}-3 \theta_{5}\right] \phi_{8}
\end{aligned}
$$

$$
\begin{aligned}
\phi_{6} \phi_{8}= & -\frac{1}{12} \theta_{2}\left[\theta_{1}^{2}-\theta_{3}\right]\left[\theta_{1}^{3}-5 \theta_{1} \theta_{3}+8 \theta_{5}\right]+\frac{1}{6}\left[\theta_{1}^{4}+\theta_{3}^{2}-6 \theta_{1}^{2} \theta_{3}+8 \theta_{1} \theta_{5}\right] \phi_{1} \\
& +\frac{1}{12}\left[\theta_{1}^{4}-6 \theta_{1}^{2} \theta_{3}+8 \theta_{1} \theta_{5}+\theta_{3}^{2}\right] \phi_{4}+\frac{1}{3} \theta_{2} \theta_{3} \phi_{6} \\
& +\frac{1}{3}\left[\theta_{1} \theta_{3}-3 \theta_{5}\right] \phi_{7}+\frac{1}{3}\left[2 \theta_{1} \theta_{3}-3 \theta_{5}\right] \phi_{8},
\end{aligned}
$$

$$
\begin{aligned}
\phi_{6} \phi_{9}= & \frac{1}{36}\left[\theta_{1}^{4} \theta_{2}^{2}-\theta_{1}^{4} \theta_{6}-6 \theta_{1}^{2} \theta_{2}^{2} \theta_{3}+18 \theta_{1} \theta_{2}^{2} \theta_{5}+6 \theta_{1} \theta_{5} \theta_{6}-9 \theta_{2}^{2} \theta_{3}^{2}-9 \theta_{3}^{2} \theta_{6}\right] \\
& -\frac{1}{9} \theta_{2}\left[\theta_{1}^{3}-6 \theta_{1} \theta_{3}+9 \theta_{5}\right] \phi_{1}-\frac{1}{18}\left[\theta_{1}^{2}-3 \theta_{3}\right] \phi_{2} \\
& +\frac{1}{3} \theta_{2}\left[\theta_{1} \theta_{3}-3 \theta_{5}\right] \phi_{4}-\frac{1}{9}\left[\theta_{1}^{2}-3 \theta_{3}\right] \phi_{5}+\frac{1}{3} \theta_{1} \theta_{6} \phi_{6} \\
& -\frac{1}{9} \theta_{2}\left[\theta_{1}^{2}-3 \theta_{3}\right] \phi_{7}-\frac{2}{9} \theta_{2}\left[\theta_{1}^{2}-3 \theta_{3}\right] \phi_{8}+\frac{1}{9} \theta_{1}^{3} \phi_{9}
\end{aligned}
$$

$$
\begin{aligned}
\phi_{6} \phi_{10}= & -\frac{1}{18} \theta_{1}\left[\theta_{1}^{3}-3 \theta_{1} \theta_{3}+3 \theta_{5}\right]\left[\theta_{2}^{2}-\theta_{6}\right]+\frac{1}{9} \theta_{2}\left[2 \theta_{1}^{3}-9 \theta_{1} \theta_{3}+9 \theta_{5}\right] \phi_{1} \\
& -\frac{1}{18}\left[\theta_{1}^{2}-3 \theta_{3}\right] \phi_{2}+\frac{1}{18}\left[\theta_{1}^{2}-3 \theta_{3}\right] \phi_{5}+\frac{1}{6} \theta_{1}\left[\theta_{2}^{2}-\theta_{6}\right] \phi_{6} \\
& +\frac{1}{18} \theta_{2}\left[\theta_{1}^{2}-3 \theta_{3}\right] \phi_{7}-\frac{1}{18} \theta_{2}\left[\theta_{1}^{2}-3 \theta_{3}\right] \phi_{8} \\
& -\frac{1}{9}\left[2 \theta_{1}^{3}-9 \theta_{1} \theta_{3}+9 \theta_{5}\right] \phi_{9}-\frac{1}{9}\left[\theta_{1}^{3}-9 \theta_{1} \theta_{3}+9 \theta_{5}\right] \phi_{10},
\end{aligned}
$$

$$
\begin{aligned}
\phi_{6} \phi_{11}= & \frac{1}{24}\left[\theta_{1}^{3} \theta_{2}^{3}-3 \theta_{1}^{3} \theta_{2} \theta_{6}-12 \theta_{1}^{3} \theta_{4}^{2}-9 \theta_{1} \theta_{2}^{3} \theta_{3}+15 \theta_{1} \theta_{2} \theta_{3} \theta_{6}+72 \theta_{1} \theta_{3} \theta_{4}^{2}+8 \theta_{2}^{3} \theta_{5}\right. \\
& \left.\quad-12 \theta_{2} \theta_{5} \theta_{6}-72 \theta_{4}^{2} \theta_{5}\right]+\frac{1}{8}\left[\theta_{1}^{2} \theta_{2}^{2}+\theta_{1}^{2} \theta_{6}+\theta_{2}^{2} \theta_{3}-3 \theta_{3} \theta_{6}\right] \phi_{1} \\
- & \frac{1}{2} \theta_{1} \theta_{2} \phi_{2}+\frac{1}{2} \phi_{3}+\frac{1}{4}\left[\theta_{2}^{3}-\theta_{2} \theta_{6}-6 \theta_{4}^{2}\right] \phi_{6} \\
+ & \frac{1}{2}\left[\theta_{1} \theta_{3}-\theta_{5}\right] \phi_{11},
\end{aligned}
$$

$$
\begin{aligned}
\phi_{7}^{2}= & \frac{1}{6}\left[\theta_{1}^{4} \theta_{6}+\theta_{1}^{2} \theta_{2}^{2} \theta_{3}-4 \theta_{1}^{2} \theta_{3} \theta_{6}+2 \theta_{1} \theta_{5} \theta_{6}-\theta_{2}^{2} \theta_{3}^{2}+\theta_{3}^{2} \theta_{6}\right] \\
& -\frac{1}{3} \theta_{2}\left[\theta_{1}^{3}-\theta_{1} \theta_{3}+2 \theta_{5}\right] \phi_{1}+\frac{1}{6}\left[3 \theta_{1}^{2}-\theta_{3}\right] \phi_{2}+\frac{2}{3} \theta_{2} \theta_{3} \phi_{7} \\
& -\frac{1}{3}\left[\theta_{1} \theta_{3}-3 \theta_{5}\right] \phi_{9},
\end{aligned}
$$

$$
\begin{aligned}
\phi_{7} \phi_{8}= & -\frac{1}{24}\left[\theta_{1}^{4} \theta_{2}^{2}+5 \theta_{1}^{4} \theta_{6}+6 \theta_{1}^{2} \theta_{2}^{2} \theta_{3}-18 \theta_{1}^{2} \theta_{3} \theta_{6}-4 \theta_{1} \theta_{2}^{2} \theta_{5}\right. \\
& \left.+4 \theta_{1} \theta_{5} \theta_{6}+\theta_{2}^{2} \theta_{3}^{2}+5 \theta_{3}^{2} \theta_{6}\right]+\frac{1}{3} \theta_{2}\left[\theta_{1}^{3}-\theta_{5}\right] \phi_{1}-\frac{1}{6}\left[3 \theta_{1}^{2}-\theta_{3}\right] \phi_{2} \\
& +\frac{1}{3} \theta_{2}\left[\theta_{1}^{3}-\theta_{5}\right] \phi_{4}-\frac{1}{6}\left[3 \theta_{1}^{2}-\theta_{3}\right] \phi_{5}+\frac{1}{3} \theta_{2} \theta_{3} \phi_{7} \\
& +\frac{1}{3} \theta_{2} \theta_{3} \phi_{8}-\frac{1}{3}\left[\theta_{1} \theta_{3}-3 \theta_{5}\right] \phi_{10},
\end{aligned}
$$


(A.49)

$$
\begin{aligned}
\phi_{7} \phi_{9}= & -\frac{1}{18}\left[\theta_{1}^{3} \theta_{2}^{3}-5 \theta_{1}^{3} \theta_{2} \theta_{6}-6 \theta_{1}^{3} \theta_{4}^{2}-8 \theta_{1} \theta_{2}^{3} \theta_{3}+20 \theta_{1} \theta_{2} \theta_{3} \theta_{6}+36 \theta_{1} \theta_{3} \theta_{4}^{2}-15 \theta_{2} \theta_{5} \theta_{6}\right. \\
& \left.+7 \theta_{2}^{3} \theta_{5}-54 \theta_{4}^{2} \theta_{5}\right]-\frac{1}{9}\left[2 \theta_{1}^{2} \theta_{2}^{2}+3 \theta_{1}^{2} \theta_{6}+3 \theta_{2}^{2} \theta_{3}-3 \theta_{3} \theta_{6}\right] \phi_{1} \\
+ & \frac{7}{9} \theta_{1} \theta_{2} \phi_{2}-\frac{1}{3} \phi_{3}-\frac{1}{9} \theta_{1}\left[\theta_{2}^{2}-6 \theta_{6}\right] \phi_{7}-\frac{1}{9} \theta_{2}\left[\theta_{1}^{2}-6 \theta_{3}\right] \phi_{9} \\
\phi_{7} \phi_{10}= & -\frac{1}{72}\left[3 \theta_{1}^{3} \theta_{2}^{3}-\theta_{1}^{3} \theta_{2} \theta_{6}+12 \theta_{1}^{3} \theta_{4}^{2}+\theta_{1} \theta_{2}^{3} \theta_{3}-7 \theta_{1} \theta_{2} \theta_{3} \theta_{6}+4 \theta_{2}^{3} \theta_{5}\right] \\
+ & \frac{1}{72}\left[7 \theta_{1}^{2} \theta_{2}^{2}-9 \theta_{2}^{2} \theta_{3}+3 \theta_{1}^{2} \theta_{6}-9 \theta_{3} \theta_{6}\right] \phi_{1}-\frac{1}{6} \theta_{1} \theta_{2} \phi_{2}+\frac{1}{6} \phi_{3} \\
+ & \frac{1}{12}\left[3 \theta_{1}^{2}-\theta_{3}\right]\left[\theta_{2}^{2}-\theta_{6}\right] \phi_{4}-\frac{2}{9} \theta_{1} \theta_{2} \phi_{5}-\frac{1}{12}\left[\theta_{2}^{3}-\theta_{2} \theta_{6}-18 \theta_{4}^{2}\right] \phi_{6} \\
+ & \frac{1}{6} \theta_{1}\left[\theta_{2}^{2}-\theta_{6}\right] \phi_{7}-\frac{1}{9} \theta_{1}\left[\theta_{2}^{2}-3 \theta_{6}\right] \phi_{8}-\frac{1}{18} \theta_{2}\left[\theta_{1}^{2}-3 \theta_{3}\right] \phi_{9} \\
- & \frac{1}{18} \theta_{2}\left[\theta_{1}^{2}-9 \theta_{3}\right] \phi_{10}-\frac{1}{6}\left[\theta_{1} \theta_{3}-3 \theta_{5}\right] \phi_{11}
\end{aligned}
$$$$
\phi_{7} \phi_{11}=-\frac{1}{36}\left[\theta_{1}^{2} \theta_{2}^{4}-4 \theta_{1}^{2} \theta_{2}^{2} \theta_{6}+3 \theta_{1}^{2} \theta_{6}^{2}-3 \theta_{2}^{4} \theta_{3}+12 \theta_{2}^{2} \theta_{3} \theta_{6}+36 \theta_{2} \theta_{3} \theta_{4}^{2}-9 \theta_{3} \theta_{6}^{2}\right]
$$$$
+\frac{1}{9} \theta_{1} \theta_{2}\left[\theta_{2}^{2}-3 \theta_{6}\right] \phi_{1}+\frac{1}{18}\left[\theta_{2}^{2}-3 \theta_{6}\right] \phi_{2}+\frac{1}{9}\left[\theta_{2}^{2}-3 \theta_{6}\right] \phi_{5}
$$$$
+\frac{1}{9} \theta_{2}^{3} \phi_{7}-\frac{1}{18}\left[5 \theta_{2}^{3}-9 \theta_{2} \theta_{6}-54 \theta_{4}^{2}\right] \phi_{8}
$$

$$
\begin{aligned}
& -\frac{1}{9} \theta_{1}\left[\theta_{2}^{2}-3 \theta_{6}\right] \phi_{9}-\frac{2}{9} \theta_{1}\left[\theta_{2}^{2}-3 \theta_{6}\right] \phi_{10}+\frac{1}{3} \theta_{2} \theta_{3} \phi_{11}, \\
& \phi_{8}^{2}=\frac{1}{6}\left[\theta_{1}^{4} \theta_{6}+2 \theta_{1}^{2} \theta_{2}^{2} \theta_{3}-5 \theta_{1}^{2} \theta_{3} \theta_{6}-3 \theta_{1} \theta_{2}^{2} \theta_{5}+5 \theta_{1} \theta_{5} \theta_{6}-\theta_{2}^{2} \theta_{3}^{2}+\theta_{3}^{2} \theta_{6}\right] \\
& -\frac{1}{3} \theta_{2}\left[\theta_{1} \theta_{3}-3 \theta_{5}\right] \phi_{1}-\frac{1}{3} \theta_{2}\left[\theta_{1}^{3}-\theta_{5}\right] \phi_{4}+\frac{1}{6}\left[3 \theta_{1}^{2}-\theta_{3}\right] \phi_{5} \\
& +\frac{2}{3} \theta_{2} \theta_{3} \phi_{8}+\frac{1}{3}\left[\theta_{1} \theta_{3}-3 \theta_{5}\right] \phi_{9}+\frac{1}{3}\left[\theta_{1} \theta_{3}-3 \theta_{5}\right] \phi_{10}, \\
& \phi_{8} \phi_{9}=\frac{1}{72}\left[\theta_{1}^{3} \theta_{2}^{3}-12 \theta_{1}^{3} \theta_{4}^{2}-27 \theta_{1}^{3} \theta_{2} \theta_{6}-13 \theta_{1} \theta_{2}^{3} \theta_{3}+43 \theta_{1} \theta_{2} \theta_{3} \theta_{6}-4 \theta_{2}^{3} \theta_{5}\right] \\
& +\frac{1}{24}\left[7 \theta_{1}^{2} \theta_{2}^{2}-\theta_{2}^{2} \theta_{3}+7 \theta_{1}^{2} \theta_{6}-5 \theta_{3} \theta_{6}\right] \phi_{1}-\frac{11}{18} \theta_{1} \theta_{2} \phi_{2} \\
& +\frac{1}{6} \phi_{3}+\frac{1}{12}\left[3 \theta_{1}^{2}-\theta_{3}\right]\left[\theta_{2}^{2}+\theta_{6}\right] \phi_{4}-\frac{4}{9} \theta_{1} \theta_{2} \phi_{5} \\
& -\frac{1}{12}\left[3 \theta_{2}^{3}-7 \theta_{2} \theta_{6}-18 \theta_{4}^{2}\right] \phi_{6}+\frac{1}{9} \theta_{1}\left[\theta_{2}^{2}-3 \theta_{6}\right] \phi_{7}+\frac{1}{9} \theta_{1} \theta_{2}^{2} \phi_{8} \\
& +\frac{1}{3} \theta_{2} \theta_{3} \phi_{9}-\frac{1}{9} \theta_{2}\left[\theta_{1}^{2}-3 \theta_{3}\right] \phi_{10}-\frac{1}{6}\left[\theta_{1} \theta_{3}-3 \theta_{5}\right] \phi_{11}, \\
& \phi_{8} \phi_{10}=-\frac{1}{72}\left[\theta_{1}^{3} \theta_{2}^{3}+\theta_{1}^{3} \theta_{2} \theta_{6}+12 \theta_{1}^{3} \theta_{4}^{2}+19 \theta_{1} \theta_{2}^{3} \theta_{3}-25 \theta_{1} \theta_{2} \theta_{3} \theta_{6}\right. \\
& \left.-144 \theta_{1} \theta_{3} \theta_{4}^{2}-20 \theta_{2}^{3} \theta_{5}+24 \theta_{2} \theta_{5} \theta_{6}+216 \theta_{4}^{2} \theta_{5}\right] \\
& +\frac{1}{72}\left[17 \theta_{1}^{2} \theta_{2}^{2}-15 \theta_{1}^{2} \theta_{6}+9 \theta_{2}^{2} \theta_{3}-3 \theta_{3} \theta_{6}\right] \phi_{1}-\frac{7}{18} \theta_{1} \theta_{2} \phi_{2} \\
& +\frac{1}{6} \phi_{3}+\frac{1}{12}\left[\theta_{2}^{3}-\theta_{2} \theta_{6}-18 \theta_{4}^{2}\right] \phi_{6}-\frac{1}{9} \theta_{1}\left[\theta_{2}^{2}-3 \theta_{6}\right] \phi_{7} \\
& +\frac{1}{6} \theta_{1}\left[\theta_{2}^{2}-\theta_{6}\right] \phi_{8}+\frac{1}{18} \theta_{2}\left[\theta_{1}^{2}-3 \theta_{3}\right] \phi_{9}+\frac{1}{3} \theta_{2} \theta_{3} \phi_{10} \\
& \text { (A.54) } \quad+\frac{1}{6}\left[\theta_{1} \theta_{3}-3 \theta_{5}\right] \phi_{11} \text {, }
\end{aligned}
$$


(A.55)

$$
\begin{aligned}
\phi_{8} \phi_{11}= & -\frac{1}{36}\left[\theta_{1}^{2} \theta_{2}^{4}-4 \theta_{1}^{2} \theta_{2}^{2} \theta_{6}+3 \theta_{1}^{2} \theta_{6}^{2}+9 \theta_{2}^{4} \theta_{3}-18 \theta_{2}^{2} \theta_{3} \theta_{6}-72 \theta_{2} \theta_{3} \theta_{4}^{2}+9 \theta_{3} \theta_{6}^{2}\right] \\
& +\frac{1}{9} \theta_{1} \theta_{2}\left[\theta_{2}^{2}-3 \theta_{6}\right] \phi_{1}-\frac{1}{9}\left[\theta_{2}^{2}-3 \theta_{6}\right] \phi_{2}-\frac{1}{18}\left[\theta_{2}^{2}-3 \theta_{6}\right] \phi_{5} \\
& +\frac{1}{18}\left[5 \theta_{2}^{3}-9 \theta_{2} \theta_{6}-54 \theta_{4}^{2}\right] \phi_{7}+\frac{1}{18}\left[7 \theta_{2}^{3}-9 \theta_{2} \theta_{6}-54 \theta_{4}^{2}\right] \phi_{8} \\
& -\frac{1}{9} \theta_{1}\left[\theta_{2}^{2}-3 \theta_{6}\right] \phi_{9}+\frac{1}{9} \theta_{1}\left[\theta_{2}^{2}-3 \theta_{6}\right] \phi_{10}+\frac{1}{3} \theta_{2} \theta_{3} \phi_{11}, \\
\phi_{9}^{2}= & \frac{1}{6}\left[\theta_{1}^{2} \theta_{2}^{2} \theta_{6}-\theta_{1}^{2} \theta_{6}^{2}-\theta_{2}^{2} \theta_{3} \theta_{6}+6 \theta_{2} \theta_{3} \theta_{4}^{2}+\theta_{3} \theta_{6}^{2}\right] \\
& -\frac{2}{3} \theta_{1}\left[\theta_{2} \theta_{6}+3 \theta_{4}^{2}\right] \phi_{1}+\frac{1}{6}\left[3 \theta_{2}^{2}-\theta_{6}\right] \phi_{2}-\frac{1}{6}\left[3 \theta_{2}^{3}-7 \theta_{2} \theta_{6}-18 \theta_{4}^{2}\right] \phi_{7} \\
& +\frac{2}{3} \theta_{1} \theta_{6} \phi_{9},
\end{aligned}
$$

$$
\begin{aligned}
\phi_{9} \phi_{10}= & -\frac{1}{24}\left[\theta_{1}^{2} \theta_{2}^{4}-12 \theta_{1}^{2} \theta_{2} \theta_{4}^{2}-\theta_{1}^{2} \theta_{6}^{2}+\theta_{2}^{4} \theta_{3}+12 \theta_{2} \theta_{3} \theta_{4}^{2}-\theta_{3} \theta_{6}^{2}\right] \\
& +\frac{1}{3} \theta_{1}\left[\theta_{2}^{3}-\theta_{2} \theta_{6}-6 \theta_{4}^{2}\right] \phi_{4}-\frac{1}{6}\left[\theta_{2}^{2}+\theta_{6}\right] \phi_{5}-\frac{1}{3}\left[\theta_{2}^{3}-2 \theta_{2} \theta_{6}-9 \theta_{4}^{2}\right] \phi_{8} \\
& +\frac{1}{6} \theta_{1}\left[\theta_{2}^{2}-\theta_{6}\right] \phi_{9}+\frac{1}{3} \theta_{1} \theta_{6} \phi_{10}-\frac{1}{6} \theta_{2}\left[\theta_{1}^{2}-3 \theta_{3}\right] \phi_{11},
\end{aligned}
$$

$$
\phi_{9} \phi_{11}=-\frac{1}{2} \theta_{1} \theta_{4}^{2}\left(\theta_{2}^{2}-\theta_{6}\right)+\frac{1}{12}\left[3 \theta_{2}^{4}-8 \theta_{2}^{2} \theta_{6}-12 \theta_{2} \theta_{4}^{2}+\theta_{6}^{2}\right] \phi_{1}
$$$$
-\frac{1}{6}\left[\theta_{2}^{2} \theta_{6}-6 \theta_{2} \theta_{4}^{2}-\theta_{6}^{2}\right] \phi_{4}+\frac{1}{3} \theta_{2} \theta_{6} \phi_{9}
$$

$$
\begin{aligned}
& -\frac{1}{6}\left[3 \theta_{2}^{3}-7 \theta_{2} \theta_{6}-18 \theta_{4}^{2}\right] \phi_{10}+\frac{1}{3} \theta_{1} \theta_{6} \phi_{11}, \\
\phi_{10}^{2}= & -\frac{1}{12}\left[12 \theta_{1}^{2} \theta_{2} \theta_{4}^{2}+\theta_{2}^{4} \theta_{3}-2 \theta_{2}^{2} \theta_{3} \theta_{6}-12 \theta_{2} \theta_{3} \theta_{4}^{2}+\theta_{3} \theta_{6}^{2}\right]+2 \theta_{1} \theta_{4}^{2} \phi_{1} \\
& -\frac{1}{6}\left[\theta_{2}^{2}-\theta_{6}\right] \phi_{2}+2 \theta_{1} \theta_{4}^{2} \phi_{4}-\frac{1}{6}\left[\theta_{2}^{2}-\theta_{6}\right] \phi_{5} \\
& +\frac{1}{6}\left[\theta_{2}^{3}-\theta_{2} \theta_{6}-18 \theta_{4}^{2}\right] \phi_{7}+\frac{1}{6}\left[\theta_{2}^{3}-\theta_{2} \theta_{6}-18 \theta_{4}^{2}\right] \phi_{8} \\
& +\frac{1}{3} \theta_{1}\left[\theta_{2}^{2}-\theta_{6}\right] \phi_{10},
\end{aligned}
$$

$$
\phi_{10} \phi_{11}=-\frac{1}{2} \theta_{1} \theta_{4}^{2}\left[\theta_{2}^{2}-\theta_{6}\right]-\frac{1}{12}\left[\theta_{2}^{4}-24 \theta_{2} \theta_{4}^{2}-\theta_{6}^{2}\right] \phi_{1}
$$$$
-\frac{1}{12}\left[\theta_{2}^{4}-2 \theta_{2}^{2} \theta_{6}-12 \theta_{2} \theta_{4}^{2}+\theta_{6}^{2}\right] \phi_{4}+\frac{1}{6}\left[\theta_{2}^{3}-\theta_{2} \theta_{6}-18 \theta_{4}^{2}\right] \phi_{9}
$$

$$
\begin{aligned}
& +\frac{1}{3}\left[\theta_{2}^{3}-\theta_{2} \theta_{6}-9 \theta_{4}^{2}\right] \phi_{10}+\frac{1}{6} \theta_{1}\left[\theta_{2}^{2}-\theta_{6}\right] \phi_{11}, \\
\phi_{11}^{2}= & -\frac{1}{8}\left[\theta_{2}^{6}-3 \theta_{2}^{4} \theta_{6}-16 \theta_{2}^{3} \theta_{4}^{2}+3 \theta_{2}^{2} \theta_{6}^{2}+24 \theta_{2} \theta_{4}^{2} \theta_{6}+72 \theta_{4}^{4}-\theta_{6}^{3}\right] \\
& +\frac{1}{2}\left[\theta_{2}^{3}-\theta_{2} \theta_{6}-6 \theta_{4}^{2}\right] \phi_{11} .
\end{aligned}
$$

Algebraic dependence syzygies for $\phi_{6}$ and $\phi_{11}$ are given by the rewriting syzygies. For the other secondary invariants, the algebraic syzygies are higher-order polynomial expressions. A representative algebraic dependence syzygy for $\phi_{4}$ is 


$$
\begin{aligned}
& 0=\left[\theta_{1}^{6} \theta_{2}^{6}-12 \theta_{1}^{6} \theta_{2}^{3} \theta_{4}^{2}-3 \theta_{1}^{6} \theta_{2}^{2} \theta_{6}^{2}-36 \theta_{1}^{6} \theta_{2} \theta_{4}^{2} \theta_{6}+36 \theta_{1}^{6} \theta_{4}^{4}-6 \theta_{1}^{6} \theta_{6}^{3}-3 \theta_{1}^{4} \theta_{2}^{6} \theta_{3}\right. \\
& -12 \theta_{1}^{4} \theta_{2}^{4} \theta_{3} \theta_{6}+36 \theta_{1}^{4} \theta_{2}^{3} \theta_{3} \theta_{4}^{2}+9 \theta_{1}^{4} \theta_{2}^{2} \theta_{3} \theta_{6}^{2}+252 \theta_{1}^{4} \theta_{2} \theta_{3} \theta_{4}^{2} \theta_{6}-324 \theta_{1}^{4} \theta_{3} \theta_{4}^{4} \\
& +54 \theta_{1}^{4} \theta_{3} \theta_{6}^{3}+4 \theta_{1}^{3} \theta_{2}^{6} \theta_{5}-24 \theta_{1}^{3} \theta_{2}^{3} \theta_{4}^{2} \theta_{5}+12 \theta_{1}^{3} \theta_{2}^{2} \theta_{5} \theta_{6}^{2}-216 \theta_{1}^{3} \theta_{2} \theta_{4}^{2} \theta_{5} \theta_{6} \\
& -48 \theta_{1}^{3} \theta_{5} \theta_{6}^{3}-9 \theta_{1}^{2} \theta_{2}^{6} \theta_{3}^{2}+36 \theta_{1}^{2} \theta_{2}^{4} \theta_{3}^{2} \theta_{6}+36 \theta_{1}^{2} \theta_{2}^{3} \theta_{3}^{2} \theta_{4}^{2}+27 \theta_{1}^{2} \theta_{2}^{2} \theta_{3}^{2} \theta_{6}^{2} \\
& -324 \theta_{1}^{2} \theta_{2} \theta_{3}^{2} \theta_{4}^{2} \theta_{6}+972 \theta_{1}^{2} \theta_{3}^{2} \theta_{4}^{4}-126 \theta_{1}^{2} \theta_{3}^{2} \theta_{6}^{3}+12 \theta_{1} \theta_{2}^{6} \theta_{3} \theta_{5}-24 \theta_{1} \theta_{2}^{4} \theta_{3} \theta_{5} \theta_{6} \\
& -216 \theta_{1} \theta_{2}^{3} \theta_{3} \theta_{4}^{2} \theta_{5}-108 \theta_{1} \theta_{2}^{2} \theta_{3} \theta_{5} \theta_{6}^{2}+648 \theta_{1} \theta_{2} \theta_{3} \theta_{4}^{2} \theta_{5} \theta_{6}+216 \theta_{1} \theta_{3} \theta_{5} \theta_{6}^{3} \\
& -9 \theta_{2}^{6} \theta_{3}^{3}+4 \theta_{2}^{6} \theta_{5}^{2}+36 \theta_{2}^{4} \theta_{3}^{3} \theta_{6}-36 \theta_{2}^{4} \theta_{5}^{2} \theta_{6}+180 \theta_{2}^{3} \theta_{3}^{3} \theta_{4}^{2} \\
& -45 \theta_{2}^{2} \theta_{3}^{3} \theta_{6}^{2}+108 \theta_{2}^{2} \theta_{5}^{2} \theta_{6}^{2}-324 \theta_{2} \theta_{3}^{3} \theta_{4}^{2} \theta_{6}-972 \theta_{3}^{3} \theta_{4}^{4}+18 \theta_{3}^{3} \theta_{6}^{3} \\
& \left.-108 \theta_{5}^{2} \theta_{6}^{3}\right]-\left[12 \theta_{1}^{5} \theta_{2}^{5}-24 \theta_{1}^{5} \theta_{2}^{3} \theta_{6}-144 \theta_{1}^{5} \theta_{2}^{2} \theta_{4}^{2}-36 \theta_{1}^{5} \theta_{2} \theta_{6}^{2}-144 \theta_{1}^{5} \theta_{4}^{2} \theta_{6}\right. \\
& -60 \theta_{1}^{3} \theta_{2}^{5} \theta_{3}+504 \theta_{1}^{3} \theta_{2}^{2} \theta_{3} \theta_{4}^{2}+252 \theta_{1}^{3} \theta_{2} \theta_{3} \theta_{6}^{2}+1080 \theta_{1}^{3} \theta_{3} \theta_{4}^{2} \theta_{6}+60 \theta_{1}^{2} \theta_{2}^{5} \theta_{5} \\
& -48 \theta_{1}^{2} \theta_{2}^{3} \theta_{5} \theta_{6}-648 \theta_{1}^{2} \theta_{2}^{2} \theta_{4}^{2} \theta_{5}-108 \theta_{1}^{2} \theta_{2} \theta_{5} \theta_{6}^{2}-648 \theta_{1}^{2} \theta_{4}^{2} \theta_{5} \theta_{6}-72 \theta_{1} \theta_{2}^{5} \theta_{3}^{2} \\
& +360 \theta_{1} \theta_{2}^{3} \theta_{3}^{2} \theta_{6}+648 \theta_{1} \theta_{2}^{2} \theta_{3}^{2} \theta_{4}^{2}-432 \theta_{1} \theta_{2} \theta_{3}^{2} \theta_{6}^{2}-1944 \theta_{1} \theta_{3}^{2} \theta_{4}^{2} \theta_{6}+60 \theta_{2}^{5} \theta_{3} \theta_{5} \\
& \left.-288 \theta_{2}^{3} \theta_{3} \theta_{5} \theta_{6}-648 \theta_{2}^{2} \theta_{3} \theta_{4}^{2} \theta_{5}+324 \theta_{2} \theta_{3} \theta_{5} \theta_{6}^{2}+1944 \theta_{3} \theta_{4}^{2} \theta_{5} \theta_{6}\right] \phi_{4} \\
& +6\left[5 \theta_{1}^{4} \theta_{2}^{4}-30 \theta_{1}^{4} \theta_{2}^{2} \theta_{6}-96 \theta_{1}^{4} \theta_{2} \theta_{4}^{2}-3 \theta_{1}^{4} \theta_{6}^{2}-54 \theta_{1}^{2} \theta_{2}^{4} \theta_{3}+108 \theta_{1}^{2} \theta_{2}^{2} \theta_{3} \theta_{6}\right. \\
& +432 \theta_{1}^{2} \theta_{2} \theta_{3} \theta_{4}^{2}+18 \theta_{1}^{2} \theta_{3} \theta_{6}^{2}+40 \theta_{1} \theta_{2}^{4} \theta_{5}-72 \theta_{1} \theta_{2}^{2} \theta_{5} \theta_{6}-432 \theta_{1} \theta_{2} \theta_{4}^{2} \theta_{5} \\
& \left.-3 \theta_{2}^{4} \theta_{3}^{2}+18 \theta_{2}^{2} \theta_{3}^{2} \theta_{6}-27 \theta_{3}^{2} \theta_{6}^{2}\right] \phi_{4}^{2}+48\left[2 \theta_{1}^{3} \theta_{2}^{3}+6 \theta_{1}^{3} \theta_{2} \theta_{6}+12 \theta_{1}^{3} \theta_{4}^{2}\right. \\
& \left.+9 \theta_{1} \theta_{2}^{3} \theta_{3}-21 \theta_{1} \theta_{2} \theta_{3} \theta_{6}-54 \theta_{1} \theta_{3} \theta_{4}^{2}-5 \theta_{2}^{3} \theta_{5}+9 \theta_{2} \theta_{5} \theta_{6}+54 \theta_{4}^{2} \theta_{5}\right] \phi_{4}^{3} \\
& \text { (A.62) }-144\left[3 \theta_{1}^{2} \theta_{2}^{2}+\theta_{1}^{2} \theta_{6}+\theta_{2}^{2} \theta_{3}-3 \theta_{3} \theta_{6}\right] \phi_{4}^{4}+576 \theta_{1} \theta_{2} \phi_{4}^{5}-288 \phi_{4}^{6} \text {. }
\end{aligned}
$$

A.6. Trigonal groups $\boldsymbol{C}_{\mathbf{3}}, \boldsymbol{S}_{\mathbf{6}}$. The rewriting syzygies are

$$
\begin{aligned}
\phi_{1}^{4} & =-\frac{1}{4} \theta_{3}^{2} \theta_{4}^{2}+\frac{1}{2} \theta_{5} \theta_{6} \phi_{1}+\frac{5}{4} \theta_{3} \theta_{4} \phi_{2}+\frac{1}{4} \theta_{3} \theta_{6} \phi_{5}+\frac{1}{4} \theta_{4} \theta_{5} \phi_{6}, \\
\phi_{1} \phi_{4} & =\phi_{11} \\
\phi_{1} \phi_{5} & =-\frac{1}{2} \theta_{4} \theta_{5}+\frac{1}{2} \theta_{3} \phi_{6}, \\
\phi_{1} \phi_{6} & =-\frac{1}{2} \theta_{3} \theta_{6}+\frac{1}{2} \theta_{4} \phi_{5}, \\
\phi_{1} \phi_{7} & =-\theta_{2} \theta_{3} \theta_{4}+\frac{1}{2} \theta_{3} \phi_{8}+\frac{1}{2} \theta_{4} \phi_{10}, \\
\phi_{1} \phi_{8} & =\frac{1}{2} \theta_{4} \phi_{7}+\frac{1}{2} \theta_{3} \phi_{9}, \\
\phi_{1} \phi_{9} & =\theta_{6} \phi_{4}+\theta_{4} \phi_{8} \\
\phi_{1} \phi_{10} & =2 \theta_{2} \theta_{3} \phi_{1}-\theta_{5} \phi_{4}+\theta_{3} \phi_{7}, \\
\phi_{4}^{2} & =\theta_{3} \theta_{4}-\phi_{2}, \\
\phi_{4} \phi_{5} & =-\theta_{2} \theta_{3} \theta_{4}-\frac{1}{2} \theta_{3} \phi_{8}+\frac{1}{2} \theta_{4} \phi_{10}, \\
\phi_{4} \phi_{6} & =\frac{1}{2} \theta_{4} \phi_{7}-\frac{1}{2} \theta_{3} \phi_{9}, \\
\phi_{4} \phi_{7} & =+\frac{1}{2} \theta_{4} \theta_{5}+\frac{1}{2} \theta_{3} \phi_{6}, \\
\phi_{4} \phi_{8} & =-\frac{1}{2} \theta_{3} \theta_{6}-\frac{1}{2} \theta_{4} \phi_{5}, \\
\phi_{4} \phi_{9} & =-\theta_{6} \phi_{1}-\theta_{4} \phi_{6}, \\
\phi_{4} \phi_{10} & =\theta_{5} \phi_{1}+2 \theta_{2} \theta_{3} \phi_{4}+\theta_{3} \phi_{5},
\end{aligned}
$$


(A.78)

$$
\begin{aligned}
\phi_{5}^{2} & =\theta_{3}^{2} \theta_{4}-\theta_{3} \phi_{2}-\theta_{5} \phi_{6}, \\
\phi_{5} \phi_{6} & =\theta_{5} \theta_{6}+2 \theta_{3} \theta_{4} \phi_{1}-2 \phi_{3}, \\
\phi_{5} \phi_{7} & =-\theta_{5} \phi_{8}+\theta_{3} \phi_{11}, \\
\phi_{5} \phi_{8} & =\theta_{2} \theta_{3} \theta_{6}-\frac{1}{2} \theta_{3} \theta_{4} \phi_{4}-\frac{1}{2} \theta_{5} \phi_{9}-\frac{1}{2} \theta_{6} \phi_{10}, \\
\phi_{5} \phi_{9} & =-\theta_{6} \phi_{7}-2 \theta_{4} \phi_{11}, \\
\phi_{5} \phi_{10} & =\theta_{3}^{2} \phi_{4}+2 \theta_{2} \theta_{3} \phi_{5}-\theta_{5} \phi_{7}, \\
\phi_{6}^{2} & =\theta_{3} \theta_{4}^{2}-\theta_{4} \phi_{2}-\theta_{6} \phi_{5}, \\
\phi_{6} \phi_{7} & =\theta_{2} \theta_{3} \theta_{6}+\frac{1}{2} \theta_{3} \theta_{4} \phi_{4}-\frac{1}{2} \theta_{5} \phi_{9}-\frac{1}{2} \theta_{6} \phi_{10}, \\
\phi_{6} \phi_{8} & =-\theta_{6} \phi_{7}-\theta_{4} \phi_{11}, \\
\phi_{6} \phi_{9} & =-\theta_{4}^{2} \phi_{4}-\theta_{6} \phi_{8}, \\
\phi_{6} \phi_{10} & =2 \theta_{2} \theta_{3} \phi_{6}-\theta_{5} \phi_{8}+2 \theta_{3} \phi_{11}, \\
\phi_{7}^{2} & =\theta_{3} \phi_{2}+\theta_{5} \phi_{6}, \\
\phi_{7} \phi_{8} & =-\theta_{5} \theta_{6}-\theta_{3} \theta_{4} \phi_{1}+2 \phi_{3}, \\
\phi_{7} \phi_{9} & =-\theta_{3} \theta_{4}^{2}+2 \theta_{4} \phi_{2}+\theta_{6} \phi_{5}, \\
\phi_{7} \phi_{10} & =\theta_{3}^{2} \phi_{1}+2 \theta_{2} \theta_{3} \phi_{7}+\theta_{5} \phi_{5}, \\
\phi_{8}^{2} & =\theta_{4} \phi_{2}+1 \theta_{6} \phi_{5}, \\
\phi_{8} \phi_{9} & =\theta_{4}^{2} \phi_{1}+\theta_{6} \phi_{6}, \\
\phi_{8} \phi_{10} & =-\theta_{3}^{2} \theta_{4}+2 \theta_{3} \phi_{2}+\theta_{5} \phi_{6}+2 \theta_{2} \theta_{3} \phi_{8}, \\
\phi_{9}^{2} & =\left[\theta_{4}^{3}-\theta_{6}^{2}\right], \\
\phi_{9} \phi_{10} & =-\theta_{5} \theta_{6}-3 \theta_{3} \theta_{4} \phi_{1}+4 \phi_{3}+2 \theta_{2} \theta_{3} \phi_{9}, \\
\phi_{10}^{2} & =-\left[4 \theta_{2}^{2} \theta_{3}^{2}-\theta_{3}^{3}+\theta_{5}^{2}\right]+4 \theta_{2} \theta_{3} \phi_{10} .
\end{aligned}
$$

A.7. Trigonal groups $C_{\mathbf{3} v}, \boldsymbol{D}_{\mathbf{3}}, \boldsymbol{D}_{\mathbf{3} \boldsymbol{d}}$. The rewriting syzygies for these groups (A.99) $\quad \phi_{1}^{4}=-\frac{1}{4} \theta_{3}^{2} \theta_{4}^{2}-\frac{1}{2} \theta_{5}\left[2 \theta_{2} \theta_{3}-\theta_{6}\right] \phi_{1}+\frac{5}{4} \theta_{3} \theta_{4} \phi_{2}-\frac{1}{4} \theta_{3} \theta_{5} \phi_{4}+\frac{1}{4} \theta_{4}\left[2 \theta_{2} \theta_{3}-\theta_{6}\right] \phi_{5}$,

(A.100) $\phi_{1} \phi_{4}=-\frac{1}{2} \theta_{4}\left[2 \theta_{2} \theta_{3}-\theta_{6}\right]+\frac{1}{2} \theta_{3} \phi_{5}$,

(A.101) $\phi_{1} \phi_{5}=\frac{1}{2} \theta_{3} \theta_{5}+\frac{1}{2} \theta_{4} \phi_{4}$,

(A.102) $\phi_{4}^{2}=\theta_{3}^{2} \theta_{4}-\theta_{3} \phi_{2}+\left(\theta_{6}-2 \theta_{2} \theta_{3}\right) \phi_{5}$,

(A.103) $\phi_{4} \phi_{5}=-\theta_{5}\left[2 \theta_{2} \theta_{3}-\theta_{6}\right]+2 \theta_{3} \theta_{4} \phi_{1}-2 \phi_{3}$,

(A.104) $\quad \phi_{5}^{2}=\theta_{3} \theta_{4}^{2}-\theta_{4} \phi_{2}+\theta_{5} \phi_{4}$.

A.8. Hexagonal groups $C_{3 h}, C_{6}, C_{6 h}$. The rewriting syzygies are

$$
\begin{aligned}
& \phi_{1}^{4}=-\frac{1}{4} \theta_{3}^{2} \theta_{4}\left[21 \theta_{4}^{3}-2 \theta_{6}\right]+\frac{1}{2} \theta_{5}\left[8 \theta_{4}^{3}-\theta_{6}\right] \phi_{1} \\
& +\frac{5}{4} \theta_{3} \theta_{4}^{2} \phi_{2}+\frac{1}{4} \theta_{3}\left[10 \theta_{4}^{3}-\theta_{6}\right] \phi_{6}+\frac{1}{4} \theta_{4}^{2} \theta_{5} \phi_{8}, \\
& \text { (A.106) } \phi_{1} \phi_{4}=2 \theta_{4} \phi_{7}-\phi_{10} \text {, } \\
& \text { (A.108) } \quad \phi_{1} \phi_{6}=-\frac{1}{2} \theta_{4}^{2} \theta_{5}+\frac{1}{2} \theta_{3} \phi_{8} \text {, }
\end{aligned}
$$$$
\text { (A.107) } \quad \phi_{1} \phi_{5}=2 \theta_{2} \theta_{3} \phi_{1}-\theta_{5} \phi_{4}+2 \theta_{3} \phi_{7} \text {, }
$$ 
(A.109) $\quad \phi_{1} \phi_{7}=-\frac{1}{2} \theta_{2} \theta_{3} \theta_{4}^{2}+\frac{1}{2} \theta_{3} \theta_{4} \phi_{4}+\frac{1}{4} \theta_{4}^{2} \phi_{5}+\frac{1}{4} \theta_{3} \phi_{9}$,

(A.110) $\phi_{1} \phi_{8}=-\frac{1}{2} \theta_{3}\left[12 \theta_{4}^{3}-\theta_{6}\right]+4 \theta_{4} \phi_{2}+\frac{1}{2} \theta_{4}^{2} \phi_{6}$,

(A.111) $\quad \phi_{1} \phi_{9}=-3 \theta_{4}^{2} \phi_{7}+2 \theta_{4} \phi_{10}+\theta_{3} \phi_{11}$,

$\phi_{1} \phi_{10}=-\frac{1}{2} \theta_{2} \theta_{3}\left[12 \theta_{4}^{3}-\theta_{6}\right]+\frac{3}{4} \theta_{3} \theta_{4}^{2} \phi_{4}$

(A.112) $+\frac{1}{4}\left[12 \theta_{4}^{3}-\theta_{6}\right] \phi_{5}+\frac{1}{2} \theta_{3} \theta_{4} \phi_{9}-\frac{1}{2} \theta_{5} \phi_{11}$,

(A.113) $\phi_{1} \phi_{11}=\frac{1}{2}\left[12 \theta_{4}^{3}-\theta_{6}\right] \phi_{4}+\frac{1}{2} \theta_{4}^{2} \phi_{9}$,

(A.114) $\quad \phi_{4}^{2}=\theta_{3} \theta_{4}^{2}-\phi_{2}$,

(A.115) $\phi_{4} \phi_{5}=-2 \theta_{3}^{2} \theta_{4}+\theta_{5} \phi_{1}+2 \theta_{2} \theta_{3} \phi_{4}+\theta_{3} \phi_{6}$,

(A.116) $\quad \phi_{4} \phi_{6}=-\theta_{2} \theta_{3} \theta_{4}^{2}+\theta_{3} \theta_{4} \phi_{4}+\frac{1}{2} \theta_{4}^{2} \phi_{5}-\frac{1}{2} \theta_{3} \phi_{9}$,

(A.117) $\quad \phi_{4} \phi_{7}=\frac{1}{4} \theta_{4}^{2} \theta_{5}-\theta_{3} \theta_{4} \phi_{1}+\frac{1}{4} \theta_{3} \phi_{8}$,

(A.118) $\quad \phi_{4} \phi_{8}=9 \theta_{4}^{2} \phi_{7}-4 \theta_{4} \phi_{10}-\theta_{3} \phi_{11}$,

(A.119) $\phi_{4} \phi_{9}=-\frac{1}{2} \theta_{3}\left[12 \theta_{4}^{3}-\theta_{6}\right]+2 \theta_{4} \phi_{2}-\frac{1}{2} \theta_{4}^{2} \phi_{6}$,

(A.120) $\phi_{4} \phi_{10}=\frac{1}{2} \theta_{4}^{3} \theta_{5}-3 \theta_{3} \theta_{4}^{2} \phi_{1}+\phi_{3}+\frac{1}{2} \theta_{3} \theta_{4} \phi_{8}$,

(A.121) $\quad \phi_{4} \phi_{11}=-\frac{1}{2}\left[6 \theta_{4}^{3}-\theta_{6}\right] \phi_{1}-\frac{1}{2} \theta_{4}^{2} \phi_{8}$

(A.122) $\quad \phi_{5}^{2}=-\left[4 \theta_{2}^{2} \theta_{3}^{2}-\theta_{3}^{3}+\theta_{5}^{2}\right]+4 \theta_{2} \theta_{3} \phi_{5}$,

(A.123) $\phi_{5} \phi_{6}=-4 \theta_{2} \theta_{3}^{2} \theta_{4}+\theta_{3}^{2} \phi_{4}+2 \theta_{3} \theta_{4} \phi_{5}+2 \theta_{2} \theta_{3} \phi_{6}-2 \theta_{5} \phi_{7}$,

(A.124) $\quad \phi_{5} \phi_{7}=-\theta_{3} \theta_{4} \theta_{5}+\frac{1}{2} \theta_{3}^{2} \phi_{1}+\frac{1}{2} \theta_{5} \phi_{6}+2 \theta_{2} \theta_{3} \phi_{7}$,

(A.125) $\quad \phi_{5} \phi_{8}=-6 \theta_{4} \theta_{5} \phi_{4}+12 \theta_{3} \theta_{4} \phi_{7}+2 \theta_{2} \theta_{3} \phi_{8}-\theta_{5} \phi_{9}-2 \theta_{3} \phi_{10}$,

(A.126) $\quad \phi_{5} \phi_{9}=3 \theta_{3}^{2} \theta_{4}^{2}-6 \theta_{4} \theta_{5} \phi_{1}+2 \theta_{3} \phi_{2}-2 \theta_{3} \theta_{4} \phi_{6}+\theta_{5} \phi_{8}+2 \theta_{2} \theta_{3} \phi_{9}$,

(A.127) $\phi_{5} \phi_{10}=-\frac{3}{2} \theta_{3} \theta_{4}^{2} \theta_{5}+3 \theta_{3}^{2} \theta_{4} \phi_{1}-\theta_{5} \phi_{2}+\theta_{4} \theta_{5} \phi_{6}-\frac{1}{2} \theta_{3}^{2} \phi_{8}+2 \theta_{2} \theta_{3} \phi_{10}$,

(A.128) $\phi_{5} \phi_{11}=-\frac{1}{2} \theta_{5}\left[10 \theta_{4}^{3}-\theta_{6}\right]-\frac{3}{2} \theta_{3} \theta_{4}^{2} \phi_{1}+2 \phi_{3}+2 \theta_{2} \theta_{3} \phi_{11}$,

(A.129) $\quad \phi_{6}^{2}=-3 \theta_{3}^{2} \theta_{4}^{2}+4 \theta_{4} \theta_{5} \phi_{1}-\theta_{3} \phi_{2}+4 \theta_{3} \theta_{4} \phi_{6}-\theta_{5} \phi_{8}$,

(A.130) $\quad \phi_{6} \phi_{7}=-\theta_{4} \theta_{5} \phi_{4}+3 \theta_{3} \theta_{4} \phi_{7}-\frac{1}{2} \theta_{5} \phi_{9}-\frac{1}{2} \theta_{3} \phi_{10}$

(A.131) $\quad \phi_{6} \phi_{8}=\theta_{5}\left[8 \theta_{4}^{3}-\theta_{6}\right]-6 \theta_{3} \theta_{4}^{2} \phi_{1}-2 \phi_{3}+4 \theta_{3} \theta_{4} \phi_{8}$,

(A.132) $\quad \phi_{6} \phi_{9}=\theta_{2} \theta_{3}\left[12 \theta_{4}^{3}-\theta_{6}\right]+\frac{3}{2} \theta_{3} \theta_{4}^{2} \phi_{4}-\frac{1}{2}\left[12 \theta_{4}^{3}-\theta_{6}\right] \phi_{5}+3 \theta_{3} \theta_{4} \phi_{9}-\theta_{5} \phi_{11}$,

(A.133) $\quad \phi_{6} \phi_{10}=-\frac{3}{2} \theta_{4}^{2} \theta_{5} \phi_{4}+\frac{3}{2} \theta_{3} \theta_{4}^{2} \phi_{7}-\theta_{4} \theta_{5} \phi_{9}+\theta_{3} \theta_{4} \phi_{10}+\frac{1}{2} \theta_{3}^{2} \phi_{11}$,

(A.134) $\phi_{6} \phi_{11}=-\left[12 \theta_{4}^{3}-\theta_{6}\right] \phi_{7}+\theta_{4}^{2} \phi_{10}+2 \theta_{3} \theta_{4} \phi_{11}$,

(A.135) $\quad \phi_{7}^{2}=-\theta_{4} \theta_{5} \phi_{1}+\frac{1}{4} \theta_{3} \phi_{2}+\frac{1}{4} \theta_{5} \phi_{8}$,

(A.136) $\quad \phi_{7} \phi_{8}=\frac{1}{2} \theta_{2} \theta_{3}\left[6 \theta_{4}^{3}-\theta_{6}\right]+\frac{9}{4} \theta_{3} \theta_{4}^{2} \phi_{4}-\frac{1}{4}\left[6 \theta_{4}^{3}-\theta_{6}\right] \phi_{5}+\theta_{3} \theta_{4} \phi_{9}-\frac{1}{2} \theta_{5} \phi_{11}$, 
(A.137)

$$
\begin{aligned}
& \phi_{7} \phi_{9}=-\frac{1}{2} \theta_{5}\left[11 \theta_{4}^{3}-\theta_{6}\right]+\frac{3}{2} \theta_{3} \theta_{4}^{2} \phi_{1}+\phi_{3}-\frac{1}{2} \theta_{3} \theta_{4} \phi_{8}, \\
& \text { (A.138) } \phi_{7} \phi_{10}=\frac{1}{8} \theta_{3}^{2}\left[12 \theta_{4}^{3}-\theta_{6}\right]-\frac{9}{4} \theta_{4}^{2} \theta_{5} \phi_{1}+\frac{1}{2} \theta_{3} \theta_{4} \phi_{2}-\frac{1}{8} \theta_{3} \theta_{4}^{2} \phi_{6}+\frac{1}{2} \theta_{4} \theta_{5} \phi_{8} \text {, } \\
& \phi_{8} \phi_{10}=-\frac{1}{2} \theta_{2} \theta_{3} \theta_{4}\left[27 \theta_{4}^{3}-2 \theta_{6}\right]+\frac{1}{2} \theta_{3}\left[18 \theta_{4}^{3}-\theta_{6}\right] \phi_{4} \\
& +\frac{1}{4} \theta_{4}\left[27 \theta_{4}^{3}-2 \theta_{6}\right] \phi_{5}+\frac{9}{4} \theta_{3} \theta_{4}^{2} \phi_{9}-3 \theta_{4} \theta_{5} \phi_{11}, \\
& \phi_{10}^{2}=\frac{1}{4} \theta_{3}^{2} \theta_{4}\left[45 \theta_{4}^{3}-4 \theta_{6}\right]-\frac{1}{2} \theta_{5}\left[18 \theta_{4}^{3}-\theta_{6}\right] \phi_{1}
\end{aligned}
$$

(A.144) $\quad \phi_{9}^{2}=-3 \theta_{4}^{2} \phi_{2}+\left[12 \theta_{4}^{3}-\theta_{6}\right] \phi_{6}$,

(A.148) $\phi_{10} \phi_{11}=-\frac{1}{4} \theta_{3} \theta_{4}^{2}\left[54 \theta_{4}^{3}-5 \theta_{6}\right]+\frac{1}{2}\left[12 \theta_{4}^{3}-\theta_{6}\right] \phi_{2}+\frac{1}{4} \theta_{4}\left[21 \theta_{4}^{3}-2 \theta_{6}\right] \phi_{6}$,

(A.149) $\quad \phi_{11}^{2}=-\frac{1}{4}\left[99 \theta_{4}^{6}-20 \theta_{4}^{3} \theta_{6}+\theta_{6}^{2}\right]$.

A.9. Hexagonal groups $D_{3 h}, C_{6 v}, D_{6}, D_{6 h}$. The rewriting syzygies are

$$
\begin{aligned}
& \phi_{1}^{4}= \frac{1}{4}\left[19 \theta_{3}^{2} \theta_{4}^{4}-2 \theta_{3}^{2} \theta_{4} \theta_{6}\right]-\frac{1}{2}\left[22 \theta_{2} \theta_{3} \theta_{4}^{3}-11 \theta_{4}^{3} \theta_{5}-2 \theta_{2} \theta_{3} \theta_{6}+\theta_{5} \theta_{6}\right] \phi_{1} \\
&+\frac{5}{4} \theta_{3} \theta_{4}^{2} \phi_{2}-\frac{1}{4} \theta_{3}\left[10 \theta_{4}^{3}-\theta_{6}\right] \phi_{4}-\frac{1}{4} \theta_{4}^{2}\left[2 \theta_{2} \theta_{3}-\theta_{5}\right] \phi_{5}, \\
&(\text { A.150) } \\
&(\text { A.151 }) \quad \phi_{1} \phi_{4}= {\left[-\theta_{2} \theta_{3} \theta_{4}^{2}+\frac{1}{2} \theta_{4}^{2} \theta_{5}\right]+\theta_{3} \theta_{4} \phi_{1}-\frac{1}{2} \theta_{3} \phi_{5}, } \\
&\left(\text { A.152) } \quad \phi_{1} \phi_{5}=\right. {\left[-4 \theta_{3} \theta_{4}^{3}+\frac{1}{2} \theta_{3} \theta_{6}\right]-2 \theta_{4} \phi_{2}-\frac{1}{2} \theta_{4}^{2} \phi_{4}, } \\
& \phi_{4}^{2}=-3 \theta_{3}^{2} \theta_{4}^{2}+2 \theta_{4}\left[2 \theta_{2} \theta_{3}-\theta_{5}\right] \phi_{1} \\
&-\theta_{3} \phi_{2}+4 \theta_{3} \theta_{4} \phi_{4}+\left[2 \theta_{2} \theta_{3}-\theta_{5}\right] \phi_{5}, \\
& \text { (A.153) } \quad \phi_{4} \phi_{5}= {\left[22 \theta_{2} \theta_{3} \theta_{4}^{3}-2 \theta_{2} \theta_{3} \theta_{6}-11 \theta_{4}^{3} \theta_{5}+\theta_{5} \theta_{6}\right] } \\
&+2 \phi_{3}+3 \theta_{3} \theta_{4} \phi_{5}, \\
& \text { (A.154) } \quad-3 \theta_{3} \theta_{4}^{4}+3 \theta_{4}^{2} \phi_{2}+\left[12 \theta_{4}^{3}-\theta_{6}\right] \phi_{4} . \\
& \text { (A.155) } \quad \phi_{5}^{2}=
\end{aligned}
$$

Acknowledgments. I thank B. Sturmfels and M. Rashid for helpful comments. 


\section{REFERENCES}

[1] E. A. ARnold, Modular algorithms for computing Gröbner bases, J. Symbolic Comput., 35 (2003), pp. 403-419.

[2] D. Bayer and M. Stillman, A theorem on refining division orders by the reverse lexicographical order, Duke Math. J., 55 (1987), pp. 321-328.

[3] T. Becker and V. Weispfenning, Gröbner Bases: A Computational Approach to Commutative Algebra, Graduate Texts in Math. 141, Springer-Verlag, New York, 1993.

[4] G. Birkhoff and S. Maclane, A Survey of Modern Algebra, 5th ed., A. K. Peters, Natick, MA, 1997.

[5] B. Buchberger, Gröbner bases: An algorithmic method in polynomial ideal theory, in Multidimensional Systems Theory, N. K. Bose, ed., D. Reidel, Boston, 1985, pp. 184-232.

[6] F. A. Сотton, Chemical Applications of Group Theory, Wiley-Interscience, New York, 1990.

[7] J.-C. FAugÉRE, A new efficient algorithm for computing Gröbner bases $\left(F_{4}\right)$, J. Pure Appl. Algebra, 139 (1999), pp. 61-88.

[8] S. Forte and M. Vianello, Symmetry classes for elasticity tensors, J. Elasticity, 43 (1996), pp. $81-108$.

[9] K. Gatermann, Computer Algebra Methods for Equivariant Dynamical Systems, SpringerVerlag, Berlin, 2000.

[10] R. Gebauer And H. M. Möller, On an installation of Buchberger's algorithm, J. Symbolic Comput., 6 (1988), pp. 275-286.

[11] A. Giovini, T. Mora, G. Niesi, L. Robbiano, and C. Traverso, "One sugar cube, please" or Selection strategies in the Buchberger algorithm, in Proceedings of the International Symposium on Algorithms and Computation, ACM, New York, 1991, pp. 49-54.

[12] T. Granlund, GNU MP: The GNU Multiple Precision Arithmetic Library, edition 4.1.2, Tech. report, Swox AB, Stockholm, Sweden, 2002.

[13] E. MAYR AND A. MEYER, The complexity of the word problem for commutative semigroups and polynomial ideals, Adv. Math., 46 (1982), pp. 305-329.

[14] G. H. Miller, An iterative Riemann solver for systems of hyperbolic conservation laws, with application to hyperelastic solid mechanics, J. Comput. Phys., 193 (2003), pp. 198-225.

[15] G. H. Miller and P. Colella, A high-order Eulerian Godunov method for elastic-plastic flow in solids, J. Comput. Phys., 167 (2001), pp. 131-176.

[16] G. H. Miller And P. Colella, A conservative three-dimensional Eulerian method for coupled fluid-solid shock capturing, J. Comput. Phys., 183 (2002), pp. 26-82.

[17] T. Molien, Über die invarianten der linearen substitutionsgruppe, Sitzungsber. Königl. Preuss. Akad. Wiss., (1897), pp. 1152-1156.

[18] F. D. Murnaghan, The Theory of Group Representations, Dover, New York, 1963.

[19] N. SloAne, Error-correcting codes and invariant theory: New applications of a nineteenthcentury technique, Amer. Math. Monthly, 84 (1977), pp. 82-107.

[20] G. F. Smith AND R. S. Rivlin, The strain-energy function for anisotropic elastic materials, Trans. Amer. Math. Soc., 88 (1958), pp. 175-193.

[21] A. J. M. Spencer, On generating functions for the number of invariants of orthogonal tensors, Mathematika, 17 (1970), pp. 275-286.

[22] A. J. M. Spencer, Theory of invariants, in Continuum Physics, Vol. 1, A. Eringen, ed., Academic Press, New York, 1971, pp. 239-353.

[23] R. Stanley, Invariants of finite groups and their applications to combinatorics, Bull. Amer. Math. Soc. (N.S.), 1 (1979), pp. 475-511.

[24] B. Sturmfels, Algorithms in Invariant Theory, Springer-Verlag, New York, 1993.

[25] H. WeyL, The Classical Groups, Their Invariants and Representations, Princeton University Press, Princeton, NJ, 1946. 